$\begin{array}{ll}\text { Research Square } & \begin{array}{l}\text { Preprints are preliminary reports that have not undergone peer review. } \\ \text { They should not be considered conclusive, used to inform clinical practice, } \\ \text { or referenced by the media as validated information. }\end{array}\end{array}$

\title{
Usefulness of Recovery services promoting personal recovery among Adults living with Severe Mental illness - A Comprehensive Integrative Review
}

Eric Badu ( $\nabla$ eric.badu@uon.edu.au )

Kwame Nkrumah University of Science and Technology College of Health Sciences https://orcid.org/0000-0002-0593-3550

Anthony Paul O'Brien

School of Nursing and Midwifery, University of Newcastle

Rebecca Mitchell

Macquarie University

\section{Research article}

Keywords: Recovery; Rehabilitation; Systematic Reviews; Mental Health; Nursing

Posted Date: April 21st, 2020

DOI: https://doi.org/10.21203/rs.3.rs-22134/v1

License: (c) (i) This work is licensed under a Creative Commons Attribution 4.0 International License. Read Full License 


\section{Abstract}

Background: There is increasing call regarding recovery services but little review have been undertaken regarding such services. This integrative review aimed to identify and synthesize evidence on the usefulness of recovery services used to promote personal recovery among adults living with severe mental illness.

Methods: A search of the published literature was conducted using: Medline, EMBASE, PsycINFO, CINAHL, Google Scholar, Web of Science, and Scopus. Mixed methods synthesis was used to analyse the data. The search was limited to papers published in English from January 2008 to January 2020 . The review integrated both qualitative and quantitative data into a single synthesis.

Results: Out of 40 included papers, $62.5 \%$ (25/40) used Quantitative data, 32.5\% used Qualitative and $5 \%$ ( $2 / 40$ ) used mixed methods. The review identified three recovery services such as integrated recovery model, vocational rehabilitation (Individual Placement Services), as well as recovery narrative photovoice and art-making services. The recovery services are useful in areas such as medication and treatment adherence, improvement in functioning, symptoms, physical health \& social behaviour, self-efficacy, economic empowerment, social inclusion, household integration and access to support services.

Conclusions: The evidence on recovery services focused largely on integrated rehabilitation and individual placement services, with a few studies implementing recovery narrative photovoice and art-making services. Mental health professionals are encouraged to implement the identified recovery services to improve the personal recovery goals of consumers.

\section{Background}

Recovery in mental health is defined as the process of restoration of functioning and well-being, improvements in quality of life of an individual diagnosed with longer-term mental health problems or emotional difficulties, using a whole system or an integrated approach $(1,2)$. The concept of recovery has been defined according to two main categories - clinical recovery (eg. service-based, or objective definition) and personal recovery (subjective) (3-8). The two forms of recovery-clinical and personal-are relevant in physical and mental conditions (9). The personal perspective considers recovery as a journey or process, whilst the clinical perspectives consider it as an outcome $(5,8)$. Personal recovery is a continuous process involving personal growth and development, improvement of symptoms, regaining control, and establishing a personally fulfilling and meaningful life, and incorporates restoration to a healthy state (3, 4 , $6,8)$. The concept also encapsulates a growing sense of agency and autonomy (8). Some studies have defined personal recovery as pursuing one's personal hopes, aspirations, personal abilities, and in the achievement of a personally acceptable quality of life $(3,4,10)$. Furthermore, some evidence has suggested that personal recovery should be given attention to provide holistic mental health services $(7,9)$. In particular, the individual's values and preferences for specific treatments or other forms of support should be central (7). Conversely, clinical recovery, which is based on a medical perspective, includes remission of symptoms or a return to normal functioning and is less holistic $(3,4,6,11)$.

A broad range of recovery services are being promoted worldwide in hospitals and community settings. Such services are operationalized as any intervention that aims to provide person-centred mental health services, thus, a multidimensional process of transformation involving positive transitions. The primary aim of recovery services is to help individuals to recover and develop some social and intellectual skills needed to live, learn and work in the community (4, 5, 8, 10). The recovery services help to improve psychological, social and cognitive functioning, social inclusion, and positive thinking of people with mental illness $(3,5-7,12)$. Despite this, past evidence has shown that several multi-level systemic and individual consumer factors continue to impede recovery services $(5-$ 8). The systemic factors are mostly associated with the strength of psychiatric facilities (patient loads, time constraints in clinical encounters), capacity of mental health professional (ancillary professionals and training support), governance or leadership of mental health services (management resistance to change and comprehensive multidisciplinary and inter-service collaboration), affordability, as well as, inclusivity of the mental health services (5-8). In addition, the individual factors include the ability to ensure early access to rehabilitation, as well as, normative life pursuits such as education, employment, sexuality, friendship, spirituality, and religious participation $(4,5,8)$.

Recently, several empirical studies regarding recovery services are being conducted to promote the personal recovery journey of consumers. The evidence has largely addressed services used to foster integrated recovery services. Despite this, only a few studies have attempted to systematically review the literature regarding recovery services for adults with Severe Mental illness (SMI). The adult with serious mental illness (SMI) may suffer from a long term condition that requires a special integrated or whole system approach to treatment. A preliminary search we conducted showed that though some existing reviews have been conducted regarding recovery, they are limited to the general mental health population and some specific services, such as individual placement services (1214). Critically, no review study has been undertaken to aggregate a synthesis of both qualitative and quantitative studies regarding recovery services that promote the personal recovery journey of Adults living with Severe Mental illness. The review, therefore, aimed to: (1) identify existing evidence on recovery services; and to (2) synthesise evidence on the relevance of the recovery services for adults living with severe mental illness.

The review findings are significant for several reasons. The evidence is expected to inform policy decision making on the wellbeing of adults with serious mental illness. The evidence is also considered to be valuable to policymakers and mental health professionals to strengthen mental health services. Finally, the evidence can guide researchers and clinicians with regard to directions for future research and further inform the training of mental health professionals and students.

\section{Methods}

\section{Methodology}

An integrative review is an approach that allows simultaneous inclusion of diverse methodologies (e.g. qualitative and quantitative data) and varied perspectives to fully understand the phenomenon of concern $(15,16)$. This review study aims to use diverse data sources to develop a holistic understanding 
of recovery services for adults with severe mental illness. This review method contributes greatly to evidence-based practice for mental health nursing. The review employed a five-stage process, including problem identification (developing and defining research question and study aim; searching literature (incorporating a comprehensive search strategy); evaluation of data (assessing for methodological quality); analysis of extracted data (data reduction, display, comparison and conclusions) and; presentation (mixed methods synthesis implications for practice, policy and research) (15).

\section{Inclusion criteria}

The review included studies that address recovery services for adults (eg 18 years and above) living with severe mental illness (eg. in-patients, out-patients, community-based residential services, home-based). The review defined individuals with serious mental illness as those with a mental, behavioural, or emotional disorder resulting in serious functional impairment, which substantially interferes with or limits one or more major life activities (17). The adult with serious mental illness were individuals with schizophrenia, bipolar disorder, mania, or psychosis that has been diagnosed by a health professional and selfreported or by proxy (18). Studies that were included targeted services such as disability support, recovery services. The study also included papers that address the effect of recovery services on the lives of people living with severe mental illness.

The review included papers of all methods and designs. Papers included used mixed methods, quantitative, or qualitative methods. The quantitative methods included quantitative randomised controlled trials, quantitative non-randomized designs (analytical cross-sectional) and quantitative descriptive studies. Also, the qualitative papers used ethnography and participatory methodology, grounded theory, phenomenology, and narrative. The review considered only studies published in the English language. Studies published from January 2008 to January 2020, were considered for inclusion in this review. This year appears as the period where researchers' increasingly attempted to research into recovery services and interventions for an adult with mental illness.

\section{Exclusion criteria}

Papers that were excluded are based purely on general health services or clinical effectiveness of a particular intervention with no connection to recovery services and mental health rehabilitation. Also, papers were excluded if they address recovery services for children and adolescents, workplace mental health issues, recovery services in stroke patients as well as in traumatic injury. The review excluded articles published prior to 2008 as well as non-English language articles. Other general exclusion criteria were systematic reviews, conference abstracts, clinical case reviews, book chapters, papers that present opinion, editorials and commentaries.

\section{Search strategy and selection procedure}

We searched seven electronic databases; EMBASE, CINAHL (EBSCO), Web of Science, Scopus, PsycINFO, Medline and Google Scholar. The searches of published articles were conducted according to the Joanna Briggs Institute (JBI) recommended guidelines for conducting systematic reviews (19). A threestage search strategy was utilised to search for information (see Table 1). An initial limited search was conducted in EMBASE and MEDLINE (Table 1). The initial search was not restricted by limiters; field, language, timespan and type of publication. We analysed the text words contained in the title and abstract and of the index terms from the initial search results (19). A second search using all identified keywords and index terms was then conducted across all remaining five databases. These searches were restricted to title, abstract and keywords due to a plethora of references obtain by the initial search. Finally, the reference lists of all identified articles were hand-searched for additional studies (19).

Table 1 Search strategy and selection procedure

\begin{tabular}{|c|c|}
\hline Stages & Search terms and keywords \\
\hline $\begin{array}{l}\text { Stage } 1 \\
\text { Initial } \\
\text { search in } \\
\text { MEDLINE } \\
\text { and } \\
\text { EMBASE }\end{array}$ & $\begin{array}{l}\text { ( Disabilit*/ OR "psychosocial disability"/ OR Adult/ or Mental Disorders/ OR mental illness/ or mental condition.mp. ) AND ( Social Support/ or "Individual } \\
\text { Support"/ or disability support.mp. OR "social inclusion"/ OR integration/ OR "community acceptance"/ or participation.mp. ) AND ( Nursing Homes/ OR } \\
\text { Residential Facilities/ or residential care.mp. or "Rehabilitation Centers"/ OR community rehabilitation.mp. OR residential program.mp. or "Residential Care" OR } \\
\text { Institutions.mp. or Residential Treatment.mp. ) AND ( "service model"/ or "service typology"/ or Rehabilitation/ or rehabilitat*.mp. or Disabled Persons/ or } \\
\text { Rehabilitation OR Vocational/ or "Psychosocial Support Systems"/ OR "Psychosocial Deprivation"/ or psychosocial.mp. ) AND ( recovery OR effectiveness/ } \\
\text { OR"Patient Reported Outcome Measures"/ OR "Treatment Outcome"/ or outcome.mp. OR "Patient Outcome Assessment"/ or functioning/ OR "quality of life"/ OR } \\
\text { coping.mp. OR "Patient-Centered Care") }\end{array}$ \\
\hline $\begin{array}{l}\begin{array}{l}\text { Stage } 2 \\
\text { (search }\end{array} \\
\text { across } \\
\text { CINAHL, } \\
\text { Web of } \\
\text { Science, } \\
\text { Scopus, } \\
\text { and } \\
\text { PsycINFO) }\end{array}$ & $\begin{array}{l}\text { ("Mental Disorders"/ OR "mental illness"/ OR "severe mental illness") AND TOPIC: (Disabilit*/ OR psychosocial disability/ OR Adult/ or Mental Disorders/ OR } \\
\text { mental illness/ or mental condition.mp.) AND TOPIC: (Social Support/ OR Individual Support/ OR disability support.mp. OR social inclusion/ OR integration/ OR } \\
\text { community acceptance/ OR participation.mp.) AND TOPIC: (Nursing Homes/ OR Residential Facilities/ OR residential care.mp. OR Rehabilitation Centers/ OR } \\
\text { community rehabilitation.mp. or community mental health rehabilitation service/ OR residential program.mp. OR Residential Care Institutions.mp. OR Residential } \\
\text { Treatment.mp.) AND TOPIC: (service model/ or service typology/ OR Rehabilitation/ or rehabilitat*.mp. or Disabled Persons/ or Rehabilitation, Vocational/ OR } \\
\text { Psychosocial Support Systems/ or Psychosocial Deprivation/ OR psychosocial.mp.) AND TOPIC: (recovery/ OR effectiveness/ OR "Patient Outcome" / OR Treatment } \\
\text { Outcome/ OR outcome.mp. OR "Patient Outcome Assessment"/ OR functioning/ OR "quality of life"/ OR coping.mp. OR "Patient-Centered") }\end{array}$ \\
\hline Stage 3 & Hand searching of the reference list \\
\hline
\end{tabular}

The selection of eligible articles adhered to the Preferred Reporting Items for Systematic Reviews and Meta-Analyses (PRISMA) (20) (Figure 1). Firstly, three authors independently screened the titles of articles that were retrieved and then approved those meeting the selection criteria. The authors reviewed all the titles and abstracts and agreed on those needing full-text screening. The first author conducted the initial screening of titles and abstracts. The second and third authors conducted the second screening of titles and abstracts of all the identified papers. The authors conducted full-text screening according to the inclusion and exclusion criteria. 
Endnote X8 (software) was used to manage the search results, screening, reviewing articles, as well as removing duplicate references. Three reviewers independently managed the data extraction process (19). The authors developed a data extraction form to handle all aspects of data extraction (Appendix 1). The data extraction form was developed according to Cochrane and the Joanna Briggs Institute (JBI) manuals (19) for conducting systematic reviews, as well as consultation with experts in methodologies and the subject area (19). The authors' extracted results of the included papers in numerical, tabular and textual format (19). The first author conducted the data extraction whilst the second and third authors conducted the second review of the extracted data. The data extraction focused on study details, (citation, year of publication, author, contact details of lead author, and funder/sponsoring organisation), publication source, methodological characteristics, study population, subject area (e.g., recovery service model, recovery concept, recovery intervention, period of project implementation, phases or component of recovery intervention, outcome or impact of intervention), as well as additional information and recommendation and other potential references to follow up.

Assessment of Methodological Quality

The authors developed a critical appraisal checklist using the Mixed Methods Appraisal Tool (MMAT) (21) and the Joanna Briggs Institute (JBI) (22) critical appraisal tool. The critical appraisal checklist was used by the authors (the first, second and third authors) to assess the methodological quality of the included papers. The critical appraisal tool was sub-divided into sections like study details, methodology (eg. categorized as qualitative, quantitative randomized controlled trials, quantitative non-randomized, including cohort study, case-control study, analytical cross-sectional, quantitative descriptive, and mixed methods) as well as overall quality score (Appendix 2). The methodological quality score was rated as low quality if the overall score was below $25 \%$, medium quality if $50 \%$ and high quality $70 \%$ and above. The scores were computed by summing the number of 'Yes' counts in each sub-section of the methodological criteria. The total score was then expressed as a percentage (21).

Data synthesis

The extracted data were analysed using a mixed-methods synthesis $(15,19)$. The mixed-methods synthesis seeks to develop an aggregated synthesis of qualitative and quantitative data (19). The process involves familiarization with the data, generating initial codes, searching for themes, reviewing themes, defining and naming themes and producing a thematic chart $(23,24)$. The authors coded the quantitative and qualitative data together. Data display matrices were developed to document all of the coded data from each extracted data (15). Alphabets and colours were assigned to each of the coded ideas. The resulting codes from quantitative and qualitative data were used to generate descriptive themes (19). The descriptive themes were categorized into global and organizing themes (see Table 3). The themes have been discussed with the concepts and theoretical constructs that explain recovery services in mental health. The background information of included papers were analysed using STATA version 15.

\section{Results}

\section{Description of retrieved papers}

The review retrieved 788 papers from all databases. Of these, 94 duplicates were removed. The titles and abstracts of 694 non-duplicate articles were screened for eligibility, after which 266 were excluded. A total of 428 full-text articles were assessed for eligibility (376 were excluded). The review extracted data from 52 full-text articles that met the eligibility. Of these, 2 articles were identified through hand searching of the reference list. Overall, 40 papers were included in the final synthesis (Figure 1). Out of the 40 papers, 38 met the criteria for high methodological quality assessment, whilst only two papers had medium quality (Table 2).

\section{Characteristics of included articles}

More than half of the papers $(23 / 40 ; 57.5 \%)$ were interventional studies. Of these, more than a third $(12 / 29 ; 43.47 \%)$ used a Randomized Controlled Trials. More than half of the included papers $(25 / 40 ; 62.5 \%)$ used Quantitative methods, $32.5 \%$ used Qualitative methods and $5 \%(2 / 40)$ used mixed methods. The participants in the included papers were mostly adults with schizophrenia and schizoaffective disorder (Table 2). About 57.5\% (23/40) of the included papers used descriptive and inferential statistics, $17.5 \%(7 / 40)$ used thematic analysis, $5 \%(2 / 40)$ each used descriptive statistics alone, grounded theory and triangulation (thematic, descriptive and inferential statistics). In addition, 27.5\% (11/40) of the included papers were studies conducted in the USA, 10\% (4/40) were studies conducted across six European centres (UK, Netherlands, Germany, Italy, Bulgaria and Switzerland), 10\% (4/40) were conducted in Sweden, 7.5\% (3/40) targeted Hong Kong and 5\% (2/40) each focused on Canada, China, South Africa and the Netherlands (see Table 1).

Table 2 Characteristics of included papers 


\begin{tabular}{|c|c|c|c|c|c|c|c|c|}
\hline Papers & Objectives & Setting & Age/Gender & Participants & Study design/Methods & $\begin{array}{c}\text { Data collection } \\
\text { instrument }\end{array}$ & Analysis & $\mathrm{Q}^{+}$ \\
\hline $\begin{array}{l}\text { Asher, } \\
\text { Hanlon (46) }\end{array}$ & $\begin{array}{l}\text { The acceptability } \\
\text { and feasibility of } \\
\text { CBR in practice, as } \\
\text { well as how CBR may } \\
\text { improve functioning } \\
\text { among people with } \\
\text { schizophrenia. }\end{array}$ & Ethiopia & $\begin{array}{l}\text { Mean age }= \\
\text { 39.5; Males } \\
\text { \& Females }\end{array}$ & Schizophrenia & $\begin{array}{c}\text { Quasi-experimental } \\
\text { design/Mixed methods }\end{array}$ & $\begin{array}{l}\text { - In-depth interviews } \\
\text { (IDIs) } \\
\text { - Discrimination and } \\
\text { Stigma Scale-12 } \\
\text { (DISC-12) } \\
\text { - Alcohol Use } \\
\text { Disorders } \\
\text { Identification Test } \\
\text { (AUDIT) } \\
\text { - Patient Health } \\
\text { Questionnaire- } \\
\text { 9(PHQ-9) } \\
\text { - Involvement } \\
\text { Evaluation } \\
\text { Questionnaire (IEQ) } \\
\text { - World Health } \\
\text { Organisation } \\
\text { Disability Assessment } \\
\text { Schedule (WHODAS) } \\
\text { 2.0 } \\
\text { - Clinical Global } \\
\text { Impression }\end{array}$ & $\begin{array}{l}\text { Thematic } \\
\text { analysis } \\
\text { and } \\
\text { Descriptive } \\
\text { statistics }\end{array}$ & $\mathrm{H}$ \\
\hline $\begin{array}{l}\text { Brooke- } \\
\text { Sumner, } \\
\text { Lund (25) }\end{array}$ & $\begin{array}{l}\text { To develop a } \\
\text { community based } \\
\text { psychosocial } \\
\text { rehabilitation } \\
\text { programme for } \\
\text { service users with } \\
\text { schizophrenia }\end{array}$ & South Africa & $\begin{array}{l}\text { Maximum }= \\
45 ; \text { Females }\end{array}$ & Schizophrenia & $\begin{array}{l}\text { Quasi-experimental } \\
\text { design/ Qualitative }\end{array}$ & $\begin{array}{l}\text { - In-depth interviews } \\
\text { (IDIs) }\end{array}$ & $\begin{array}{l}\text { Thematic } \\
\text { analysis }\end{array}$ & $\mathrm{H}$ \\
\hline $\begin{array}{l}\text { Brooke- } \\
\text { Sumner, } \\
\text { Selohilwe } \\
(26)\end{array}$ & $\begin{array}{l}\text { Investigated a non- } \\
\text { specialist delivered } \\
\text { programme for } \\
\text { psychosocial } \\
\text { rehabilitation for } \\
\text { service users with } \\
\text { schizophrenia in a } \\
\text { low-resource South } \\
\text { African setting }\end{array}$ & South Africa & $\begin{array}{l}\text { Range }=21- \\
44 ; \text { Males } \\
\& \text { Females }\end{array}$ & Schizophrenia & $\begin{array}{c}\text { Quasi-experimental } \\
\text { design/Mixed methods }\end{array}$ & $\begin{array}{l}\text { - In-depth interviews } \\
\text { (IDIs) } \\
\text { - Brief Psychiatric } \\
\text { Rating Scale (BPRS) } \\
\text { - Clinician- } \\
\text { administered scale } \\
\text { - World Health } \\
\text { Organization } \\
\text { Disability Assessment } \\
\text { Scale (WHODAS) } \\
\text { - Stigma of Mental } \\
\text { Illness Inventory } \\
\text { (ISMI) }\end{array}$ & $\begin{array}{l}\text { Thematic } \\
\text { analysis } \\
\text { and } \\
\text { Inferential } \\
\text { statistics }\end{array}$ & $\mathrm{H}$ \\
\hline $\begin{array}{l}\text { Browne and } \\
\text { Waghorn } \\
\text { (53) }\end{array}$ & $\begin{array}{l}\text { to retrospectively } \\
\text { assess the } \\
\text { implementation of } \\
\text { IPS practices and } \\
\text { youth employment } \\
\text { outcomes }\end{array}$ & $\begin{array}{l}\text { New } \\
\text { Zealand }\end{array}$ & $\begin{array}{l}\text { Age range of } \\
16-25 \text { years; } \\
\text { Males } \\
\text { \&Females }\end{array}$ & $\begin{array}{c}\text { Affective (including } \\
\text { comorbid anxiety), Bipolar } \\
\text { affective disorder, } \\
\text { Generalized anxiety disorder }\end{array}$ & $\begin{array}{l}\text { Observational design } \\
\text { (Retrospective case } \\
\text { study)/Quantitative }\end{array}$ & - Case review & $\begin{array}{l}\text { Descriptive } \\
\text { statistics }\end{array}$ & $\mathrm{M}$ \\
\hline $\begin{array}{l}\text { Burns, } \\
\text { White (35) }\end{array}$ & $\begin{array}{l}\text { The acceptability } \\
\text { and effectiveness of } \\
\text { IPS in Europe }\end{array}$ & $\begin{array}{l}\text { Five } \\
\text { European } \\
\text { countries }\end{array}$ & $\begin{array}{l}\text { Mean age }= \\
38 \text { years }\end{array}$ & $\begin{array}{c}\text { schizophrenia or } \\
\text { schizoaffective disorder } \\
\text { bipolar disorder }\end{array}$ & $\begin{array}{c}\text { Randomized } \\
\text { controlled } \\
\text { trial/Quantitative }\end{array}$ & - Questionnaire & $\begin{array}{l}\text { Inferential } \\
\text { statistics }\end{array}$ & High \\
\hline $\begin{array}{l}\text { Catty, } \\
\text { Lissouba } \\
(51)\end{array}$ & $\begin{array}{l}\text { Determine which } \\
\text { patients with severe } \\
\text { mental illness do } \\
\text { well in vocational } \\
\text { services and which }\end{array}$ & $\begin{array}{l}\text { Six } \\
\text { European } \\
\text { centres }\end{array}$ & $\begin{array}{l}\text { Range (18 to } \\
\text { local } \\
\text { retirement } \\
\text { age);Males } \\
\text { \&Females }\end{array}$ & Schizophrenia & $\begin{array}{c}\text { Randomised } \\
\text { controlled trial/ } \\
\text { Quantitative }\end{array}$ & $\begin{array}{l}\text { - Global Assessment of } \\
\text { Functioning - } \\
\text { Symptoms (GAF-S) } \\
\text { and Disability (GAF- } \\
\text { D) }\end{array}$ & $\begin{array}{l}\text { Inferential } \\
\text { statistics }\end{array}$ & $\mathrm{H}$ \\
\hline
\end{tabular}

Page 5/22 


\begin{tabular}{|c|c|c|c|c|c|c|c|c|}
\hline & $\begin{array}{l}\text { process and service } \\
\text { factors are } \\
\text { associated with } \\
\text { better outcomes }\end{array}$ & & & & & $\begin{array}{l}\text { - Positive and } \\
\text { Negative Syndrome } \\
\text { Scale } \\
\text { - Hospital Anxiety and } \\
\text { Depression Scale } \\
\text { - Groningen Social } \\
\text { Disability Schedule } \\
\text { - Lancashire Quality of } \\
\text { Life Profile - } \\
\text { European Version } \\
\text { - Rosenberg Self- } \\
\text { Esteem Scale } \\
\text { - Camberwell } \\
\text { Assessment of Need - } \\
\text { European short } \\
\text { version }\end{array}$ & & \\
\hline $\begin{array}{l}\text { Chang, } \\
\text { Chen (27) }\end{array}$ & $\begin{array}{l}\text { Investigated the } \\
\text { effect of a music } \\
\text { creation group } \\
\text { programme on the } \\
\text { anxiety, self-esteem, } \\
\text { and quality of life of } \\
\text { patients with SMI }\end{array}$ & Taiwan & $\begin{array}{l}\text { Range }(20- \\
65) ; \text { Males } \\
\text { \&Females }\end{array}$ & $\begin{array}{c}\text { Schizophrenia or affective } \\
\text { disorders }\end{array}$ & $\begin{array}{l}\text { Quasi-experimental } \\
\text { design/ Quantitative }\end{array}$ & $\begin{array}{l}\text { - Demographic data } \\
\text { - Hamilton Anxiety } \\
\text { Rating Scale (HAM- } \\
\text { A) } \\
\text { - Rosenberg Self- } \\
\text { Esteem Scale (RSES) } \\
\text { - World Health } \\
\text { Organization Quality } \\
\text { of Life-BREF } \\
\text { (WHOQOL-BREF) }\end{array}$ & $\begin{array}{l}\text { Inferential } \\
\text { statistics }\end{array}$ & $\mathrm{H}$ \\
\hline $\begin{array}{l}\text { Chiu, Ho } \\
\text { (28) }\end{array}$ & $\begin{array}{l}\text { To test empirically } \\
\text { the substance Abuse } \\
\text { and mental Health } \\
\text { Services } \\
\text { administration } \\
\text { (SAMHSA) recovery } \\
\text { model }\end{array}$ & Hong Kong & $\begin{array}{l}\text { Mean age = } \\
41.6 \\
\text { (Average) } \\
\text { Males } \\
\text { \&Females }\end{array}$ & $\begin{array}{l}\text { Schizophrenia spectrum } \\
\text { disorder }\end{array}$ & $\begin{array}{c}\text { Cross- } \\
\text { sectional/Quantitative }\end{array}$ & $\begin{array}{l}\text { - Internalized Stigma } \\
\text { of Mental Illness } \\
\text { scale (ISMI) } \\
\text { - Resilience Scale (RS) } \\
\text { - Making Decision } \\
\text { Empowerment scale } \\
\text { (MDES) } \\
\text { - Exercise of Self-Care } \\
\text { Agency Scale } \\
\text { - Mastery Scale (MS) } \\
\text { - Adult State Hope } \\
\text { Scale (ASHS) } \\
\text { - Health Care Climate } \\
\text { Questionnaire } \\
\text { (HCCQ) } \\
\text { - Recovery Attitude } \\
\text { Questionnaire (RAQ- } \\
\text { 7) } \\
\text { - Medical Outcome } \\
\text { Study Social Support } \\
\text { Survey-Chinese } \\
\text { version (EISS-MOS- } \\
\text { SSS-C) } \\
\text { - Schizophrenia } \\
\text { Quality of Life Scale } \\
\text { (SQLS) } \\
\text { - Multidimensional } \\
\text { Scale of Perceived } \\
\text { Social Support- } \\
\text { Chinese version } \\
\text { (MSPSS-C) } \\
\text { - World Health } \\
\text { Organization } \\
\text { Spirituality Religion } \\
\text { and Personal Belief } \\
\text { Scale-Hong Kong } \\
\text { version (WHO- } \\
\text { SRPBHK) } \\
\text { M }\end{array}$ & $\begin{array}{l}\text { Inferential } \\
\text { statistics }\end{array}$ & $\mathrm{H}$ \\
\hline $\begin{array}{l}\text { Clements } \\
\text { (56) }\end{array}$ & $\begin{array}{l}\text { To pilot a PAR and } \\
\text { photovoice project, } \\
\text { to facilitate } \\
\text { discussions about } \\
\text { recovery based on } \\
\text { personal and local } \\
\text { experience }\end{array}$ & Canada & & & $\begin{array}{l}\text { Participatory action } \\
\text { research/Qualitative } \\
\text { (eg. photovoice) }\end{array}$ & $\begin{array}{l}\text { Photo/text pieces, other } \\
\text { 'readers' or audiences }\end{array}$ & $\begin{array}{l}\text { Recovery } \\
\text { Photo } \\
\text { Gallery }\end{array}$ & $\mathrm{H}$ \\
\hline
\end{tabular}




\begin{tabular}{|c|c|c|c|c|c|c|c|c|}
\hline $\begin{array}{l}\text { Crain, } \\
\text { Penhale } \\
\text { (31) }\end{array}$ & $\begin{array}{l}\text { Application of IPS in } \\
\text { a Canadian } \\
\text { community mental } \\
\text { health team through } \\
\text { the study of a } \\
\text { competitively } \\
\text { employed individual } \\
\text { and his support } \\
\text { network. }\end{array}$ & Canada & $\begin{array}{l}\text { Mean age = } \\
42 \\
\text { years/Males }\end{array}$ & Schizophrenia & $\begin{array}{l}\text { Instrumental case } \\
\text { study/Qualitative }\end{array}$ & $\begin{array}{l}\text { - In-depth interviews } \\
\text { (IDIs) }\end{array}$ & $\begin{array}{l}\text { Thematic } \\
\text { analysis }\end{array}$ & $\mathrm{H}$ \\
\hline $\begin{array}{l}\text { De Heer- } \\
\text { Wunderink, } \\
\text { Visser (32) }\end{array}$ & $\begin{array}{l}\text { Investigated levels of } \\
\text { social inclusion } \\
\text { among service users } \\
\text { of two types of } \\
\text { psychiatric } \\
\text { community housing } \\
\text { programs in the } \\
\text { Netherlands }\end{array}$ & Netherlands & $\begin{array}{l}\text { Mean age } 44 \\
\text { years / Males } \\
\& \text { Females }\end{array}$ & $\begin{array}{l}\text { Schizophrenia, Anxiety or } \\
\text { depression, Personality } \\
\text { disorder }\end{array}$ & $\begin{array}{c}\text { Cross-sectional } \\
\text { Design/Quantitative }\end{array}$ & $\begin{array}{l}\text { Health of the Nation } \\
\text { Outcome Scales } \\
\text { (HoNOS) } \\
\text { - Camberwell } \\
\text { Assessment of Need } \\
\text { Short Appraisal Scale } \\
\text { (CANSAS) }\end{array}$ & $\begin{array}{l}\text { Descriptive } \\
\text { and } \\
\text { Inferential } \\
\text { statistics }\end{array}$ & $\mathrm{H}$ \\
\hline $\begin{array}{l}\text { Fenner, } \\
\text { Ryan (57) }\end{array}$ & $\begin{array}{l}\text { analysed what } \\
\text { consumers and staff } \\
\text { reported at the end } \\
\text { of the project }\end{array}$ & Samoa & & & $\begin{array}{c}\text { Interpretive } \\
\text { Phenomenological } \\
\text { Design/ Qualitative }\end{array}$ & $\begin{array}{l}\text { - Focus group } \\
\text { Discussion }\end{array}$ & IPA & $\mathrm{H}$ \\
\hline $\begin{array}{l}\text { Harpaz- } \\
\text { Rotem, } \\
\text { Rosenheck } \\
\text { (33) }\end{array}$ & $\begin{array}{l}\text { Observational data } \\
\text { comparing 1-year } \\
\text { clinical outcomes } \\
\text { among women who } \\
\text { received RT services } \\
\text { and those who did } \\
\text { not. }\end{array}$ & USA & & $\begin{array}{l}\text { Psychiatric/substance abuse } \\
\text { problems }\end{array}$ & $\begin{array}{l}\text { Quasi-experimental } \\
\text { design/ Quantitative }\end{array}$ & $\begin{array}{l}\text { - Self-report interview } \\
\text { - psychiatric, Alcohol, } \\
\text { and Drug composite } \\
\text { scales } \\
\text { - Short Form Health } \\
\text { Survey (SF-12) } \\
\text { - Symptom Checklist- } \\
30 \text { (SCL) } \\
\text { - Posttraumatic Stress } \\
\text { Disorder (PTSD) } \\
\text { Symptom Checklist } \\
\text { (PCL) }\end{array}$ & $\begin{array}{l}\text { Descriptive } \\
\& \\
\text { Inferential } \\
\text { statistics }\end{array}$ & $\mathrm{H}$ \\
\hline $\begin{array}{l}\text { Hultqvist, } \\
\text { Markstrom } \\
\text { (36) }\end{array}$ & $\begin{array}{l}\text { Comparing users of } \\
\text { two approaches to } \\
\text { psychosocial } \\
\text { rehabilitation in } \\
\text { Sweden, community- } \\
\text { based mental health } \\
\text { day centres (DCs) } \\
\text { and clubhouses }\end{array}$ & Sweden & $\begin{array}{l}\text { Mean age }= \\
48.7 \text { years; } \\
\text { Males } \\
\& \text { Females }\end{array}$ & $\begin{array}{c}\text { Psychoses, Mood and anxiety } \\
\text { disorders, } \\
\text { Autism/neuropsychiatric } \\
\text { disorders }\end{array}$ & $\begin{array}{l}\text { Quasi-experimental } \\
\text { design/Quantitative }\end{array}$ & $\begin{array}{l}\text { - Manchester Short } \\
\text { Assessment of } \\
\text { Quality of Life } \\
\text { (MANSA) } \\
\text { - Self-esteem } \\
\text { Rosenberg } \\
\text { - Global Assessment of } \\
\text { Functioning (GAF) } \\
\text { - MOS 36-item short- } \\
\text { form health survey } \\
\text { - Social Interaction } \\
\text { (ISSI) } \\
\text { - Satisfaction with } \\
\text { daily occupations } \\
\text { (SDO) } \\
\text { - Swedish version of } \\
\text { the CSQ-8 } \\
\text { - The EPM-DC } \\
\text { - Socio-demographic } \\
\text { and clinical factors }\end{array}$ & $\begin{array}{l}\text { Descriptive } \\
\& \\
\text { Inferential } \\
\text { statistics }\end{array}$ & \\
\hline $\begin{array}{l}\text { Hultqvist, } \\
\text { Markström } \\
\text { (63) }\end{array}$ & $\begin{array}{l}\text { compared DC and } \\
\text { clubhouses, } \\
\text { concerning the } \\
\text { users' perceptions of } \\
\text { unit and programme }\end{array}$ & Sweden & $\begin{array}{l}\text { Mean age }= \\
48.7 \text { years; } \\
\text { Males } \\
\& \text { Females }\end{array}$ & $\begin{array}{c}\text { Psychoses, Mood and anxiety } \\
\text { disorders, } \\
\text { Autism/neuropsychiatric } \\
\text { disorders }\end{array}$ & $\begin{array}{l}\text { Combined cross- } \\
\text { sectional and } \\
\text { longitudinal } \\
\text { comparative study/ } \\
\text { Quantitative }\end{array}$ & $\begin{array}{l}\text { - Evaluation of } \\
\text { Perceived Meaning } \\
\text { in Day Centres (EPM- } \\
\text { DC) }\end{array}$ & $\begin{array}{l}\text { Descriptive } \\
\& \\
\text { Inferential } \\
\text { statistics }\end{array}$ & \\
\hline
\end{tabular}

Page $7 / 22$ 


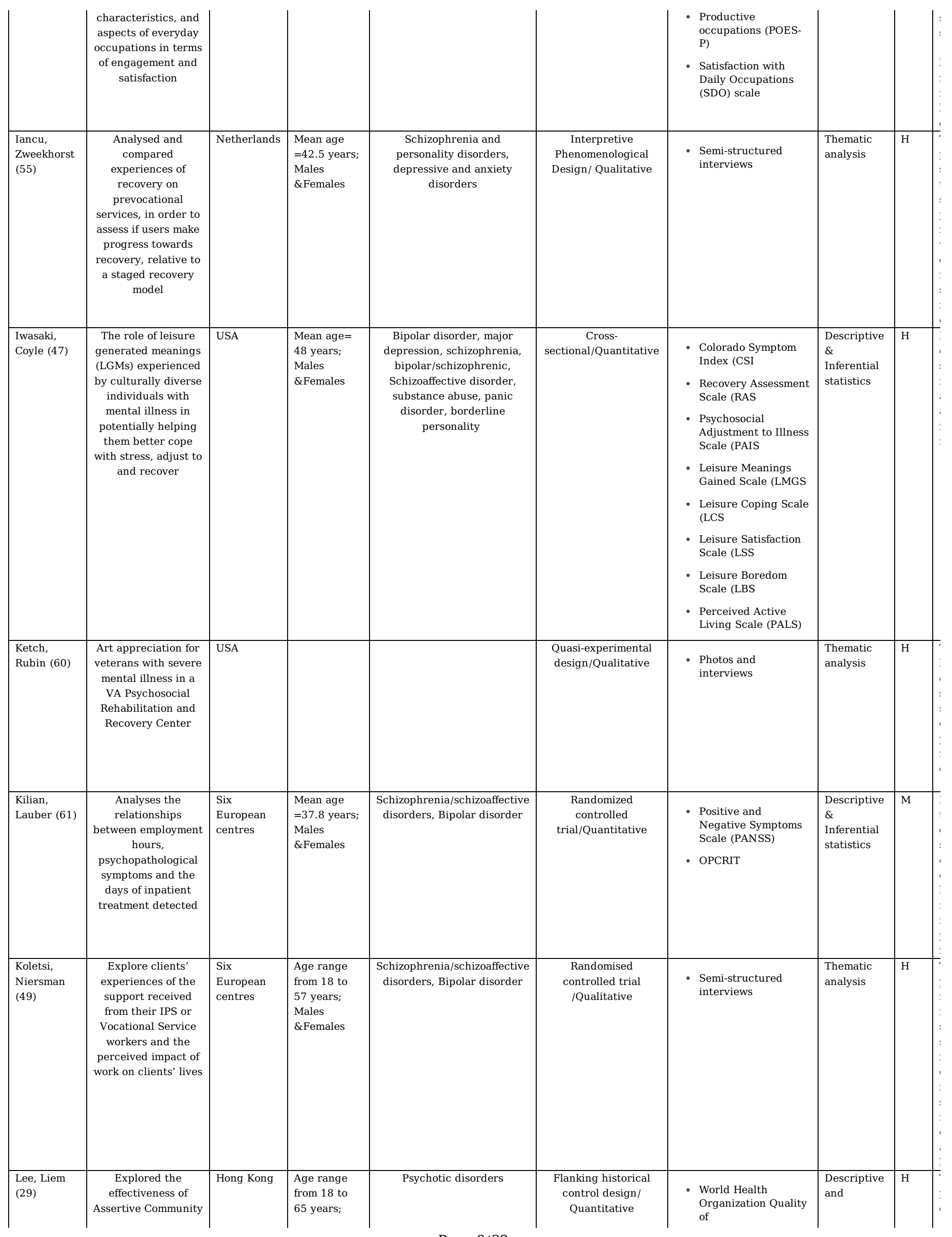

Page $8 / 22$ 


\begin{tabular}{|c|c|c|c|c|c|c|c|c|}
\hline & $\begin{array}{l}\text { Treatment (ACT) for } \\
\text { severely ill mental } \\
\text { patients during a } \\
\text { period of rapid } \\
\text { deinstitutionalization } \\
\text { in Hong Kong }\end{array}$ & & $\begin{array}{l}\text { Males } \\
\& \text { Females }\end{array}$ & & & $\begin{array}{l}\text { Life(WHOQOL-) Hong } \\
\text { Kong Chinese } \\
\text { Version } \\
\text { - Brief Psychiatric } \\
\text { Rating Scale (BPRS) } \\
\text { - Clinical Data } \\
\text { Analysis and } \\
\text { Reporting System } \\
\text { (CDARS) }\end{array}$ & $\begin{array}{l}\text { Inferential } \\
\text { Statistics }\end{array}$ & \\
\hline $\begin{array}{l}\text { Lindstrom, } \\
\text { Hariz (34) }\end{array}$ & $\begin{array}{l}\text { To evaluate clients' } \\
\text { activities of daily } \\
\text { living (ADL) ability } \\
\text { and health factors } \\
\text { outcomes following } \\
\text { their participation in } \\
\text { occupation-centred } \\
\text { interventions in } \\
\text { home and } \\
\text { community settings }\end{array}$ & Sweden & $\begin{array}{l}\text { Mean age }= \\
48 \text { years; } \\
\text { Males } \\
\& \text { Females }\end{array}$ & $\begin{array}{c}\text { Schizophrenia, } \\
\text { Schizoaffective disorder, } \\
\text { Bipolar disorder, Asperger } \\
\text { syndrome, Obsessive } \\
\text { compulsive disorder }\end{array}$ & $\begin{array}{l}\text { Quasi-experimental } \\
\text { design / Quantitative }\end{array}$ & $\begin{array}{l}\text { - Goal Attainment } \\
\text { Scaling (GAS) } \\
\text { - Assessment of Motor } \\
\text { and Process Skills } \\
\text { (AMPS) } \\
\text { - Assessment of Social } \\
\text { Interaction (Swedish } \\
\text { version BSI-II) } \\
\text { - Satisfaction with } \\
\text { Daily Occupations } \\
\text { (SDO) } \\
\text { - ADL-taxonomy } \\
\text { - Symptom Checklist- } \\
90 \text { (SCL-90) }\end{array}$ & $\begin{array}{l}\text { Descriptive } \\
\text { and } \\
\text { Inferential } \\
\text { Statistics }\end{array}$ & $\mathrm{H}$ \\
\hline $\begin{array}{l}\text { Lopez- } \\
\text { Navarro, } \\
\text { Del Canto } \\
\text { (45) }\end{array}$ & $\begin{array}{l}\text { The effectiveness of } \\
\text { group mindfulness- } \\
\text { based intervention } \\
\text { (MBI) in patients } \\
\text { diagnosed with } \\
\text { severe mental illness }\end{array}$ & $\begin{array}{l}\text { Balearic } \\
\text { Islands }\end{array}$ & $\begin{array}{l}\text { Mean age } \\
=38.44 \text { years }\end{array}$ & $\begin{array}{c}\text { Schizophrenia, Bipolar } \\
\text { disorder, Delusional disorder }\end{array}$ & $\begin{array}{c}\text { Randomised } \\
\text { controlled trial/ } \\
\text { Quantitative }\end{array}$ & $\begin{array}{l}\text { - World Health } \\
\text { Organization Quality } \\
\text { of Life-BREF } \\
\text { (WHOQOL-BREF) } \\
\text { - Positive and } \\
\text { Negative Syndrome } \\
\text { Scale (PANSS) } \\
\text { - Mindfulness } \\
\text { Attention Awareness } \\
\text { Scale (MAAS) }\end{array}$ & $\begin{array}{l}\text { Descriptive } \\
\text { and } \\
\text { Inferential } \\
\text { Statistics }\end{array}$ & $\mathrm{H}$ \\
\hline Luk (38) & $\begin{array}{l}\text { Investigate the long- } \\
\text { term effects of a } \\
\text { holistic care } \\
\text { programme for the } \\
\text { rehabilitation of } \\
\text { persons with serious } \\
\text { mental illness }\end{array}$ & Hong Kong & $\begin{array}{l}\text { Mostly aged } \\
35 \text { or above; } \\
\text { Males } \\
\& \text { Females }\end{array}$ & $\begin{array}{l}\text { Schizophrenia, Manic } \\
\text { Depressive, Depression }\end{array}$ & $\begin{array}{c}\text { Cross- } \\
\text { sectional/Quantitative }\end{array}$ & $\begin{array}{l}\text { - World Health } \\
\text { Organization Quality } \\
\text { of Life Measure } \\
\text { (WHOQOL-BREF(HK) } \\
\text { - General Health } \\
\text { Questionnaire (GHQ) } \\
\text { - Rosenberg Self- } \\
\text { esteem Scale } \\
\text { (ESTEEM) } \\
\text { - Social Support } \\
\text { Questionnaire-6 } \\
\text { (SSQ-6) } \\
\text { - Purpose in Life } \\
\text { Questionnaire (PIL) } \\
\text { - Hopelessness Scale } \\
\text { (HOPE) } \\
\text { - SSQ-Satisfaction }\end{array}$ & $\begin{array}{l}\text { Descriptive } \\
\text { and } \\
\text { Inferential } \\
\text { Statistics }\end{array}$ & $\mathrm{H}$ \\
\hline $\begin{array}{l}\text { Malinovsky, } \\
\text { Lehrer (39) }\end{array}$ & $\begin{array}{c}\text { Evaluate the } \\
\text { effectiveness of a } \\
\text { recovery-oriented } \\
\text { transformation } \\
\text { carried out by a } \\
\text { large, private, not- } \\
\text { for-profit psychiatric } \\
\text { rehabilitation } \\
\text { organization serving } \\
\text { individuals with SM }\end{array}$ & USA & $\begin{array}{l}\text { Mean age } \\
=46.42 \\
\text { years; Males } \\
\& \text { Females }\end{array}$ & $\begin{array}{c}\text { Schizophrenia, Mood } \\
\text { disorder (unipolar/bipolar), } \\
\text { Other psychotic disorder }\end{array}$ & $\begin{array}{c}\text { Longitudinal } \\
\text { study/Quantitative }\end{array}$ & $\begin{array}{l}\text { - Multnomah } \\
\text { Community Ability } \\
\text { Scale-Revised } \\
\text { Clinician Rated } \\
\text { (MCAS-R) } \\
\text { - Self-Report (MCAS- } \\
\text { SR) } \\
\text { - Competency } \\
\text { Assessment } \\
\text { Instrument (CAI) } \\
\text { - State Hope Scale } \\
\text { (SHS) } \\
\text { - Client Version (WAI- } \\
\text { C) and Therapist } \\
\text { Version (WAI-T) }\end{array}$ & $\begin{array}{l}\text { Descriptive } \\
\text { and } \\
\text { Inferential } \\
\text { Statistics }\end{array}$ & $\overline{\mathrm{H}}$ \\
\hline
\end{tabular}




\begin{tabular}{|c|c|c|c|c|c|c|c|c|}
\hline $\begin{array}{l}\text { Mizock, } \\
\text { Russinova } \\
(58)\end{array}$ & $\begin{array}{c}\text { Describe the } \\
\text { development and } \\
\text { feasibility of the } \\
\text { Recovery Narrative } \\
\text { Photovoice } \\
\text { intervention }\end{array}$ & USA & & Serious mental illnesses & $\begin{array}{c}\text { Community-based } \\
\text { participatory } \\
\text { research/ Quantitative }\end{array}$ & $\begin{array}{l}\text { - Ryff Scale of } \\
\text { Psychological Well- } \\
\text { Being } \\
\text { - Empowerment Scale } \\
\text { - Community } \\
\text { Integration Measure }\end{array}$ & $\begin{array}{l}\text { Descriptive } \\
\text { Statistics }\end{array}$ & \\
\hline $\begin{array}{l}\text { Mizock, } \\
\text { Russinova } \\
(59)\end{array}$ & $\begin{array}{c}\text { Explore the meaning } \\
\text { of recovery for } \\
\text { individuals with } \\
\text { serious mental } \\
\text { illness }\end{array}$ & USA & & Serious mental illness & $\begin{array}{c}\text { Community-based } \\
\text { participatory } \\
\text { research/ Qualitative }\end{array}$ & $\begin{array}{l}\text { - Photos and archival } \\
\text { data }\end{array}$ & $\begin{array}{l}\text { Thematic } \\
\text { analysis }\end{array}$ & $\mathrm{H}$ \\
\hline $\begin{array}{l}\text { Panczak and } \\
\text { Pietkiewicz } \\
\text { (52) }\end{array}$ & $\begin{array}{c}\text { Explore personal } \\
\text { experiences of } \\
\text { people employed in } \\
\text { Vocational } \\
\text { Development } \\
\text { Centers }\end{array}$ & Poland & $\begin{array}{l}\text { Age range } \\
\text { from 28-58 } \\
\text { years; Males } \\
\& \text { Females }\end{array}$ & $\begin{array}{c}\text { Schizophrenia spectrum } \\
\text { disorders }\end{array}$ & $\begin{array}{c}\text { Interpretative } \\
\text { phenomenological } \\
\text { design/ Qualitative }\end{array}$ & $\begin{array}{l}\text { - Semi-structured } \\
\text { interviews }\end{array}$ & $\begin{array}{l}\text { Consecutive } \\
\text { analytical }\end{array}$ & $\mathrm{H}$ \\
\hline $\begin{array}{l}\text { Raeburn, } \\
\text { Schmied } \\
(62)\end{array}$ & $\begin{array}{c}\text { Explore how } \\
\text { recovery practices } \\
\text { are implemented in a } \\
\text { psychosocial } \\
\text { clubhouse }\end{array}$ & Australia & $\begin{array}{l}\text { Mean age= } \\
47 \text { years; } \\
\text { Males } \\
\& \text { Females }\end{array}$ & $\begin{array}{c}\text { Schizophrenia, bipolar } \\
\text { disorder or schizoaffective } \\
\text { disorder }\end{array}$ & $\begin{array}{l}\text { Case study/ } \\
\text { Qualitative }\end{array}$ & $\begin{array}{l}\text { In-depth interviews } \\
\text { (IDIs) } \\
\text { - Observations - } \\
\text { Spradley's field note } \\
\text { domains, and the } \\
\text { Recovery and } \\
\text { Promotion Fidelity } \\
\text { Scale (RPFS) }\end{array}$ & $\begin{array}{l}\text { Theoretical } \\
\text { thematic } \\
\text { analysis }\end{array}$ & $\mathrm{H}$ \\
\hline $\begin{array}{l}\text { Salyers, } \\
\text { McGuire } \\
(40)\end{array}$ & $\begin{array}{c}\text { To rigorously test } \\
\text { Illness Management } \\
\text { and Recovery (IMR) } \\
\text { against an active } \\
\text { control group in a } \\
\text { sample that included } \\
\text { veterans. }\end{array}$ & USA & $\begin{array}{l}\text { Mean age } \\
=47.7 \text { years; } \\
\text { Males } \\
\text { \&Females }\end{array}$ & $\begin{array}{c}\text { Schizophrenia, } \\
\text { Schizoaffective disorder }\end{array}$ & $\begin{array}{c}\text { Randomized } \\
\text { controlled trial/ } \\
\text { Quantitative }\end{array}$ & $\begin{array}{l}\text { - Structured Clinical } \\
\text { Interview for DSM-IV } \\
\text { - Positive and } \\
\text { Negative Syndrome } \\
\text { Scale (PANSS) } \\
\text { - Quality of Life Scale } \\
\text { (QLS) } \\
\text { - Patient Activation } \\
\text { Measure } \\
\text { - Morisky Scale } \\
\text { - Recovery Assessment } \\
\text { Scale (RAS) } \\
\text { - State Hope Scale }\end{array}$ & $\begin{array}{l}\text { Descriptive } \\
\text { and } \\
\text { Inferential } \\
\text { Statistics }\end{array}$ & $\mathrm{H}$ \\
\hline $\begin{array}{l}\text { Svanberg, } \\
\text { Gumley (54) }\end{array}$ & $\begin{array}{l}\text { Explore the } \\
\text { experience of } \\
\text { recovery from } \\
\text { mental illness in the } \\
\text { context of two } \\
\text { emerging social } \\
\text { firms }\end{array}$ & Scotland & $\begin{array}{l}\text { Age range } \\
\text { from 19-64 } \\
\text { years; Males } \\
\& \text { Females }\end{array}$ & $\begin{array}{c}\text { Bipolar disorder, depression, } \\
\text { psychosis, anxiety, } \\
\text { addictions }\end{array}$ & $\begin{array}{c}\text { Social constructionist } \\
\text { (Grounded theory)/ } \\
\text { Qualitative }\end{array}$ & $\begin{array}{l}\text { - Open-ended } \\
\text { interview questions }\end{array}$ & $\begin{array}{l}\text { Thematic } \\
\text { analysis }\end{array}$ & $\mathrm{H}$ \\
\hline $\begin{array}{l}\text { Swildens, } \\
\text { van } \\
\text { Busschbach } \\
(64)\end{array}$ & $\begin{array}{l}\text { Investigate the effect } \\
\text { of the Boston } \\
\text { Psychiatric } \\
\text { Rehabilitation (PR) } \\
\text { Approach on } \\
\text { attainment of } \\
\text { personal } \\
\text { rehabilitation goals, } \\
\text { social functioning, } \\
\text { empowerment, needs } \\
\text { for care, and quality } \\
\text { of life in people with } \\
\text { severe mental illness } \\
\text { (SMI) in the } \\
\text { Netherlands }\end{array}$ & Netherlands & $\begin{array}{c}\text { Mostly aged } \\
41 \text { or above; } \\
\text { Males } \\
\& \text { Females }\end{array}$ & $\begin{array}{c}\text { Schizophrenia or } \\
\text { schizoaffective disorder, } \\
\text { Bipolar disorder, Depressive } \\
\text { or anxiety disorder, } \\
\text { Personality, Addiction, } \\
\text { Cognitive disorder }\end{array}$ & $\begin{array}{c}\text { Randomized } \\
\text { Controlled Trial/ } \\
\text { Quantitative }\end{array}$ & $\begin{array}{l}\text { - Self-report Social } \\
\text { Functioning Scale } \\
\text { - Camberwell } \\
\text { Assessment of Need } \\
\text { Short Appraisal } \\
\text { Schedule } \\
\text { - WHOQOL-BREF } \\
\text { - Personal } \\
\text { Empowerment Scale } \\
\text { - BPRS-Extended } \\
\text { version } \\
\text { - GAF-symptoms and } \\
\text { disabilities } \\
\text { - Client Socio- } \\
\text { demographic and } \\
\text { Service Receipt }\end{array}$ & $\begin{array}{l}\text { Descriptive } \\
\text { and } \\
\text { Inferential } \\
\text { Statistics }\end{array}$ & $\mathrm{H}$ \\
\hline
\end{tabular}

Page 10/22 


\begin{tabular}{|c|c|c|c|c|}
\hline & & & & $m=$ \\
\hline$=$ & 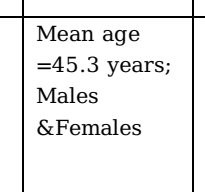 & $=$ & $=$ & $=$ \\
\hline$=$ & 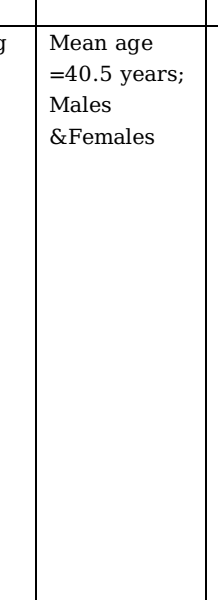 & & $=$ & 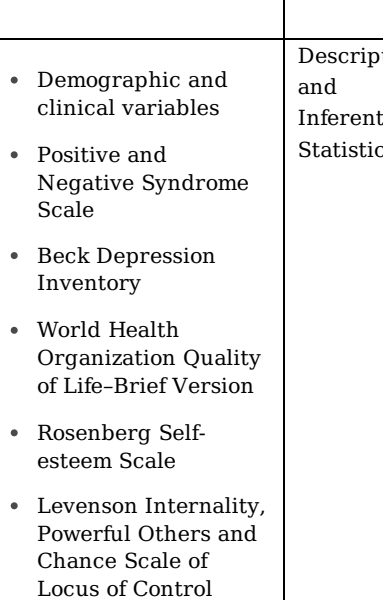 \\
\hline $\begin{array}{l}= \\
\equiv \\
\equiv \\
\equiv\end{array}$ & 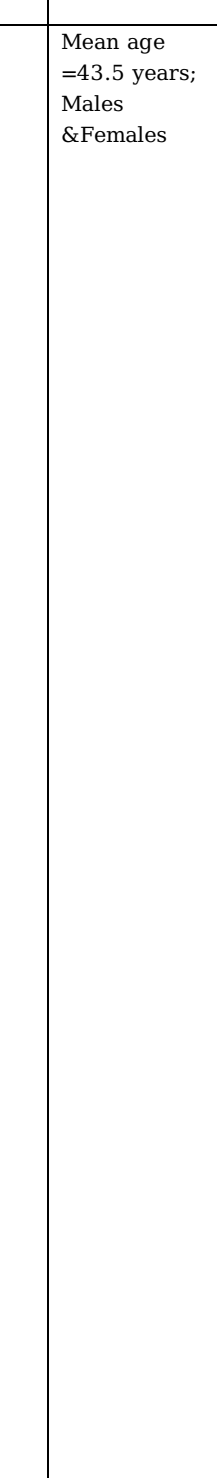 & 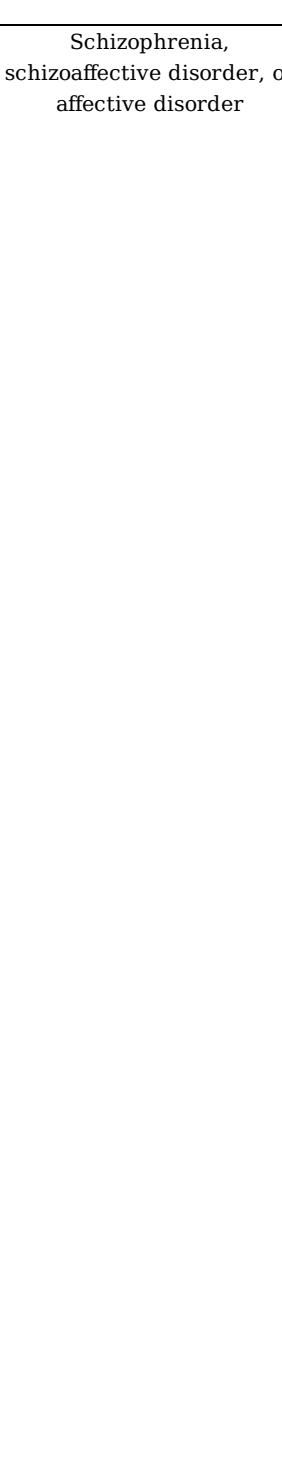 & $=5$ & 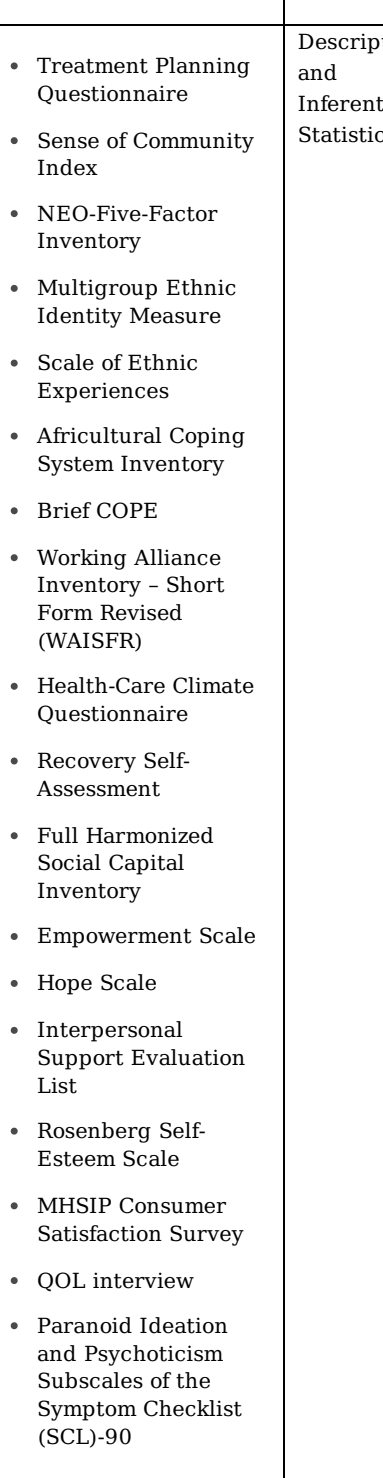 \\
\hline
\end{tabular}

Page $11 / 22$ 


\begin{tabular}{|c|c|c|c|c|c|c|c|c|}
\hline & & & & & & $\begin{array}{l}\text { - SCL-90 anxiety } \\
\text { dimension } \\
\text { - Global Assessment of } \\
\text { Functioning - } \\
\text { Modified Version }\end{array}$ & & \\
\hline $\begin{array}{l}\text { Twamley, } \\
\text { Vella (48) }\end{array}$ & $\begin{array}{l}\text { To evaluate the } \\
\text { efficacy of supported } \\
\text { employment for } \\
\text { middle-aged or older } \\
\text { people with } \\
\text { schizophrenia. }\end{array}$ & USA & $\begin{array}{l}\text { Mean age } \\
=51 \text { years; } \\
\text { Males } \\
\text { \&Females }\end{array}$ & $\begin{array}{c}\text { Schizophrenia or } \\
\text { schizoaffective disorder aged }\end{array}$ & $\begin{array}{l}\text { Randomized } \\
\text { controlled trial/ } \\
\text { Quantitative }\end{array}$ & $\begin{array}{l}\text { - UCSD Performance- } \\
\text { Based Skills } \\
\text { Assessment (UPSA) } \\
\text { - Positive and } \\
\text { Negative Syndrome } \\
\text { Scale (PANSS) } \\
\text { - Hamilton Rating } \\
\text { Scale for Depression }\end{array}$ & $\begin{array}{l}\text { Descriptive } \\
\text { and } \\
\text { Inferential } \\
\text { Statistics }\end{array}$ & $\mathrm{H}$ \\
\hline $\begin{array}{l}\text { Waghorn, } \\
\text { Dias (50) }\end{array}$ & $\begin{array}{l}\text { This investigation } \\
\text { compared the utility } \\
\text { of two approaches to } \\
\text { measuring the } \\
\text { effectiveness of a } \\
\text { supported } \\
\text { employment } \\
\text { programme }\end{array}$ & Australia & $\begin{array}{l}\text { Mean age } \\
=34.1 \text { years; } \\
\text { Males } \\
\text { \&Females }\end{array}$ & $\begin{array}{c}\text { Schizophrenia, } \\
\text { schizoaffective disorder, } \\
\text { schizophreniform disorders, } \\
\text { bipolar affective disorder, } \\
\text { major depression and anxiety } \\
\text { disorders }\end{array}$ & $\begin{array}{c}\text { Non-randomised trial/ } \\
\text { Quantitative }\end{array}$ & $\begin{array}{l}\text { - IPS fidelity scale } \\
\text { - Socially Valued Role } \\
\text { Classification Scale } \\
\text { (SRCS) } \\
\text { - Scale for the } \\
\text { Assessment of } \\
\text { Positive Symptoms } \\
\text { (SAPS) } \\
\text { - Scale for the } \\
\text { Assessment of } \\
\text { Negative Symptoms } \\
\text { (SANS) } \\
\text { - Demographic } \\
\text { information }\end{array}$ & $\begin{array}{l}\text { Descriptive } \\
\text { and } \\
\text { Inferential } \\
\text { Statistics }\end{array}$ & $\mathrm{H}$ \\
\hline $\begin{array}{l}\text { Whitley, } \\
\text { Harris (42) }\end{array}$ & $\begin{array}{c}\text { Explore and } \\
\text { elucidate whether } \\
\text { components of these } \\
\text { communities } \\
\text { appeared to assist } \\
\text { recovery from the } \\
\text { point of view of } \\
\text { consumers, and if so } \\
\text { which were the most } \\
\text { important factors }\end{array}$ & USA & & & $\begin{array}{c}\text { Grounded theory } \\
\text { approach/ Qualitative }\end{array}$ & $\begin{array}{l}\text { - Focus groups } \\
\text { - Observations }\end{array}$ & $\begin{array}{l}\text { grounded } \\
\text { theory } \\
\text { approach }\end{array}$ & $\mathrm{H}$ \\
\hline $\begin{array}{l}\text { Zemore and } \\
\text { Kaskutas } \\
(44)\end{array}$ & $\begin{array}{l}\text { explores whether } \\
\text { services received } \\
\text { differed by program } \\
\text { modality (i.e., day } \\
\text { hospital vs. } \\
\text { residential) }\end{array}$ & USA & $\begin{array}{l}\text { Adults aged } \\
\geq 18 \text { years; } \\
\text { Males } \\
\text { \&Females }\end{array}$ & $\begin{array}{l}\text { Alcohol dependent only Drug } \\
\text { dependent only } \\
\text { Alcohol and drug dependent }\end{array}$ & $\begin{array}{c}\text { Randomized } \\
\text { controlled trial/ } \\
\text { Quantitative }\end{array}$ & $\begin{array}{l}\text { - Treatment Services } \\
\text { Review (TSR } \\
\text { - Demographics and } \\
\text { other covariates }\end{array}$ & $\begin{array}{l}\text { Descriptive } \\
\text { and } \\
\text { Inferential } \\
\text { Statistics }\end{array}$ & $\mathrm{H}$ \\
\hline $\begin{array}{l}\text { Zhou, Zhou } \\
\text { (43) }\end{array}$ & $\begin{array}{l}\text { Effectiveness of the } \\
\text { rehabilitation } \\
\text { services provided at } \\
\text { the 'Sunshine Soul } \\
\text { Park' on the } \\
\text { psychotic symptoms } \\
\text { and social } \\
\text { functioning of } \\
\text { individuals with } \\
\text { schizophrenia }\end{array}$ & China & $\begin{array}{l}\text { Mean age } \\
=39.2 \text { years; } \\
\text { Males } \\
\text { \&Females }\end{array}$ & Schizophrenia & $\begin{array}{l}\text { Non- Randomize } \\
\text { Controlled trial/ } \\
\text { Quantitative }\end{array}$ & $\begin{array}{l}\text { - } \text { PANSS } \\
\text { - Quality of Life } \\
\text { Inventory-74 } \\
\text { (GQOLI-74) } \\
\text { - Social Disability } \\
\text { Screening Schedule } \\
\text { (SDSS) } \\
\text { - Insight and } \\
\text { Treatment Attitude } \\
\text { Questionnaire (ITAQ) }\end{array}$ & $\begin{array}{l}\text { Descriptive } \\
\text { and } \\
\text { Inferential } \\
\text { Statistics }\end{array}$ & $\mathrm{H}$ \\
\hline
\end{tabular}

$\mathrm{A}^{+}$represent the average, minimum or maximum age of participants $\mathrm{Q}^{++}$represent the quality assessment score for the papers (" $\mathrm{H}$ " indicate high score whilst " $\mathrm{M}$ " indicate medium score) 
The review identified five environments where recovery services are implemented. The environment of implementing recovery services were community-based interventions, residential facility setting and services via psychiatric day hospital/primary health care settings (25-30) (Table 3). Four papers suggested that recovery services can be offered through home-based cares (31-34) and a day centre structure programme (35-37).

Table 3 Descriptive thematic network

\begin{tabular}{|c|c|c|c|}
\hline Global themes & Organizing themes & $\mathbf{N}$ & Papers \\
\hline \multirow{5}{*}{$\begin{array}{l}\text { Environment for } \\
\text { implementing } \\
\text { recovery } \\
\text { services }\end{array}$} & $\begin{array}{l}\text { Community-Based } \\
\text { Intervention }\end{array}$ & 22 & 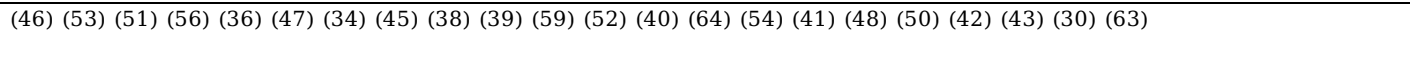 \\
\hline & $\begin{array}{l}\text { Residential } \\
\text { facilities }\end{array}$ & 7 & $(53)(35)(51)(57)(33)(60)(44)$ \\
\hline & $\begin{array}{l}\text { Home-Based Care } \\
\text { or services }\end{array}$ & 4 & $(31)(32)(33)(34)$ \\
\hline & $\begin{array}{l}\text { Day Center } \\
\text { Structured } \\
\text { Programme } \\
\end{array}$ & 3 & $(35)(36)(37)$ \\
\hline & $\begin{array}{l}\text { Psychiatric day } \\
\text { hospital/primary } \\
\text { services }\end{array}$ & 6 & $(25)(26)(27)(28)(29)(30)$ \\
\hline \multirow[t]{3}{*}{$\begin{array}{l}\text { Philosophy of } \\
\text { Recovery } \\
\text { services }\end{array}$} & $\begin{array}{l}\text { Integrated } \\
\text { recovery service } \\
\text { model }\end{array}$ & 17 & $(46)(47)(29)(25)(26)(45)(38)(39)(40)(41)(44)(43)(42)(32)(37)(34)(27)$ \\
\hline & $\begin{array}{l}\text { Vocational } \\
\text { Rehabilitation }\end{array}$ & 18 & (53) (35) (51) (31) (32) (48) (50) (52) (49) (61) (54) (64) (37) (55) (36) (63) (30) \\
\hline & $\begin{array}{l}\text { Recovery } \\
\text { Narrative } \\
\text { Photovoice and Art } \\
\text { making }\end{array}$ & 5 & (56) (59) (58) (57) (60) \\
\hline \multirow{9}{*}{$\begin{array}{l}\text { Usefulness of } \\
\text { recovery } \\
\text { services }\end{array}$} & $\begin{array}{l}\text { Psychiatric } \\
\text { medication }\end{array}$ & 10 & $(46)(25)(33)(31)(61)(29)(39)(43)(40)(44)$ \\
\hline & $\begin{array}{l}\text { Improving } \\
\text { functionality }\end{array}$ & 14 & $(46)(26)(33)(55)(36)(60)(49)(29)(34)(52)(40)(64)(43)(39)$ \\
\hline & Reduce symptoms & 11 & $(27)(28)(33)(61)(29)(34)(45)(40)(43)(30)(25)$ \\
\hline & $\begin{array}{l}\text { Improving physical } \\
\text { health \& Social } \\
\text { Behaviour }\end{array}$ & 7 & $(46)(26)(33)(34)(45)(39)$ \\
\hline & $\begin{array}{l}\text { Economic } \\
\text { empowerment }\end{array}$ & 19 & 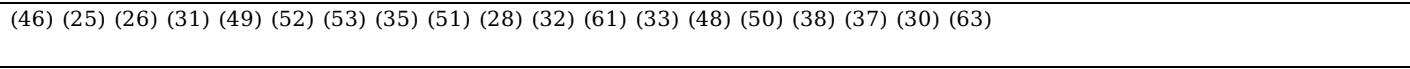 \\
\hline & $\begin{array}{l}\text { Household } \\
\text { integration }\end{array}$ & 3 & $(46)(26)(33)$ \\
\hline & $\begin{array}{l}\text { Social inclusion } \\
\text { (Community } \\
\text { integration) }\end{array}$ & 27 & $(46)(25)(26)(27)(28)(56)(31)(32)(57)(55)(36)(60)(49)(29)(34)(52)(62)(54)(64)(37)(41)(42)(44)(43)(63)(30)(58)$ \\
\hline & Social support & 7 & $(28)(55)(42)(59)(33)(31)(32)$ \\
\hline & Self-efficacy & 21 & 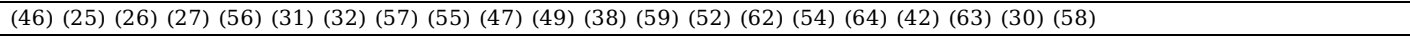 \\
\hline
\end{tabular}

\section{Philosophy of Recovery services}

\section{An integrated recovery service model}

In this review, integrated recovery service model is described as any services that seek to promote and support restoration, 'remediation and reconnection. The concept employs both an overarching, inherently collaborative and integrated approach to mental health services. Sixteen of the included papers described the integrated recovery services model used to achieve recovery for adults with severe mental illness (Table 3). Most of the papers suggested that integrated recovery service model can be delivered through illness management (38-44), mindfulness-based interventions (45), task-sharing or shifting approach (25, $26)$, home visits concept $(29,46,47)$, active leisure or recreational activities $(32,37)$ and music therapy $(27)$ (see Table 4). The reviewed papers highlighted that the integrated recovery services generally aim at developing independent living skills, improve quality of life, community mobilisation (39), reducing inpatient and crisis services, adhering to treatment and setting meaningful goals towards recovery $(40,41,46)$.

The integrated recovery service model can be offered through training sessions (e.g. hours, days and weekly for several months) $(25-27,29,40,45)$. For instance, past study regarding integrated recovery model was enhanced with mindfulness group therapy sessions which were run for 60 minutes throughout 26 weeks (45) (see Table 4). Conversely, music-creation therapy used as recovery services were run for 32 weekly sessions, with a duration of 90 min for each session (27). Generally, the activities of recovery service model covers components such as cognitive behaviour therapy techniques, psychoeducation, relapse prevention, social and coping skills training (meals, guidance in activities of daily living, role-playing, hobby groups) $(38,40,46)$, adherence support, family therapy, crisis management, household contribution and understanding medication $(25,29,46)$. More specifically, Tjornstrand, Bejerholm $(37)$ recommended that active leisure implemented as recovery services can take several activities, including playing sports, the opportunity to play games, eat, and socialize, embarking on excursions and relaxation (see Table 4).

Two studies concluded that conventional rehabilitation services can incorporate additional innovative interventions aimed to achieve recovery for consumers $(27,38)$. For instance, Luk $(38)$ recommended the inclusion of spiritual intervention (a form of hymn singing, Bible reading, personal sharing and intercessions) into the conventional rehabilitation services. Similarly, Chang, Chen (27) recommended the use of music-creation therapy as a recovery service 
for adults with SMI. The recovery services are delivered by different mental health professionals, including a clinical psychologist, community health workers, psychiatrists, occupational therapists, social workers and counsellor $(25,26,29,38,47)$. Some studies further suggested that non-specialist are sometimes trained to deliver recovery services, through task-sharing or task-shifting approach. Some of the non-specialist professionals are auxiliary social workers (25, 38).

\section{Vocational Rehabilitation (Individual Placement Services)}

Eighteen of the included papers recommended several vocational rehabilitation programmes used to promote recovery among consumers (see Table 3 ). These are Individual Placement and Support (IPS) (30-32, 35, 48-51), supported employment enterprises, sheltered employment (30, 32, 52, 53), conventional vocational rehabilitation (48) and social firms $(52,54)$.

Most of the included papers described the process of implementing individual placement and support. The papers suggested that the IPS is implemented through phases such as initial vocational assessments (eg. to identify clients' personal strengths and work skills), job searching (eg. searching job sites, applying for work online and accompany clients to interviews), individual job development (eg. intensive supervision), work performance monitoring, support for employers and continuing post-employment support for clients $(31,35,48,51,53)$. In addition, some papers recommended that Individual placement support workers receive training and regular supervision to provide effective services $(50,51)$ (see Table 4$)$. The IPS employment can take several forms, including services(eg. cleaning, gardening, catering, clerical and administrative work) $(30,37)$, training clients for labour market, agricultural production and creative projects (eg. painting, drawing, sculpture, ceramics and textiles, assembly lines, carpentry shops, computer repair centres, bicycle repair shops woodworking and furniture making) $(54,55)$.

\section{Recovery Narrative Photovoice, Art-making and exhibition}

Five of the included papers recommended photovoice, art-making and exhibition as interventions used to construct recovery (56-60). The photovoice, artmaking and exhibition are used to achieve recovery, empowerment, community integration $(56,58,59)$ and share difficult experiences non-verbally $(57)$. The intervention aims to explore, document and share ideas about recovery. It involves the construction of text with photographs through the exhibition and large group discussion $(56,58,59)$. More importantly, the intervention helps to avoid the stigma that is associated with the conventional process of delivering mental health services (60). For instance, Clements (56) suggested that readers or audience of photovoice interventions become part of the construction of the meaning of recovery. The intervention allows people with serious mental illness to communicate their needs and ideas to the public, as well as to policymakers.

The photovoice, art and exhibition intervention are delivered through weekly class sessions and community outings (58-60) (Table 3). The content of the class sessions focuses on writing exercises, psychoeducational handouts, and activities on how to construct empowering narratives of recovery and stigmatization $(58,59)$ (see Table 4). In addition, Ketch, Rubin (60) suggested that the class session begins with sharing previous experiences or knowledge about artmaking. The final outcome of the photovoice, art and exhibition intervention are documented through the final recovery photo gallery or text pieces, art shows or public exhibition, creative arts (eg. painting, ceramics, silk screening, mosaics) and displays of art prints $(56,57,59,60)$.

Table 4 Mode of delivering Recovery oriented rehabilitation services 


\begin{tabular}{|c|c|c|c|}
\hline $\begin{array}{l}\text { Recovery } \\
\text { oriented } \\
\text { rehabilitation } \\
\text { services }\end{array}$ & Intervention & How to deliver the intervention & Process or Outcome \\
\hline \multirow{5}{*}{$\begin{array}{l}\text { Integrated } \\
\text { Rehabilitation }\end{array}$} & Mindfulness-based interventions & $\begin{array}{l}\text { - Mindfulness group therapy sessions (Training for hourly, days } \\
\text { and weekly) } \\
\text { - Meetings for consumers and family members }\end{array}$ & $\begin{array}{l}\text { Improving functioning, symptoms and quality of } \\
\text { life }\end{array}$ \\
\hline & Task-sharing or shifting approach & $\begin{array}{l}\text { - Participatory training for non-specialist to provide mental health } \\
\text { service in communities } \\
\text { - Refer adults living with severe mental illness to primary health } \\
\text { care and specialist services }\end{array}$ & $\begin{array}{l}\text { Increase access to psychiatric medication, } \\
\text { improve physical health \& social Behaviour, self- } \\
\text { efficacy }\end{array}$ \\
\hline & Home visits concept & $\begin{array}{l}\text { Weekly home visits by mental health professionals to deliver } \\
\text { mental health education, advocacy, community outreach and } \\
\text { community orientation } \\
\text { - Adults living with severe mental illness and family members are } \\
\text { thought about where and how to buy medication } \\
\text { - Reminding consumers and families to attend follow-up }\end{array}$ & $\begin{array}{l}\text { Household integration, improving self-care or } \\
\text { practical skills }\end{array}$ \\
\hline & Music-creation therapy & $\begin{array}{l}\text { 90-min music-creation group activity organized weekly for } 32 \\
\text { consecutive weeks }\end{array}$ & $\begin{array}{l}\text { Community acceptance, inclusion, } \\
\text { empowerment }\end{array}$ \\
\hline & Everyday life rehabilitation & $\begin{array}{l}\text { - Weekly meeting for approximately } 1 \text { to } 2 \text { hours } \\
\text { - Set personal goals such as regular walks, weekly sauna bathing, } \\
\text { social interaction with friends, initiating small talk with women, } \\
\text { eating lunch in a restaurant, healthy cooking, and taking control } \\
\text { of one's own pocket money }\end{array}$ & Self-efficacy (self-esteem or self-confidence) \\
\hline \multirow[t]{2}{*}{$\begin{array}{l}\text { Vocational } \\
\text { Rehabilitation }\end{array}$} & $\begin{array}{l}\text { Individual Placement and Support } \\
\text { (IPS) }\end{array}$ & $\begin{array}{l}\text { - Initial vocational assessments } \\
\text { - Job searching } \\
\text { - Individual job development } \\
\text { - Training and regular supervision } \\
\text { - Work performance monitoring } \\
\text { - Support for employers and continuing post-employment support }\end{array}$ & $\begin{array}{l}\text { Returning to open employment, gaining } \\
\text { competitive employment, economic } \\
\text { empowerment, gaining financial literacy skills, } \\
\text { financial independence and stability }\end{array}$ \\
\hline & $\begin{array}{l}\text { Conventional vocational } \\
\text { rehabilitation (eg. Sheltered } \\
\text { employment, Supported } \\
\text { employment enterprises and Social } \\
\text { firms) }\end{array}$ & & Economic empowerment \\
\hline \multirow[t]{2}{*}{$\begin{array}{l}\text { Narrative } \\
\text { Photovoice } \\
\text { and Art } \\
\text { making }\end{array}$} & Photovoice & $\begin{array}{l}\text { - Taking of photographs from daily life and constructing text, } \\
\text { exhibition and large group discussion } \\
\text { - Exploring, documenting and sharing ideas about recovery } \\
\text { - Weekly class sessions and community outings and exercises } \\
\text { on construction of recovery }\end{array}$ & $\begin{array}{l}\text { Empowerment, community integration, hope, } \\
\text { progress in recovery }\end{array}$ \\
\hline & Art making and exhibition & $\begin{array}{l}\text { - Weekly art appreciation class took place for a several months } \\
\text { - Art Appreciation class includes both classroom sessions and } \\
\text { community outings }\end{array}$ & $\begin{array}{l}\text { Development of new skills, community } \\
\text { integration }\end{array}$ \\
\hline
\end{tabular}


Ten of the included papers highlighted that recovery services has helped to improve clinical outcomes of adults living with SMI (25, 29, 31, 33, 39, 40, 43, 44, $46,61)$. The services specifically increase access to psychiatric medication, antipsychotic medication adherence, decrease relapse, improve knowledge and illness management, as well as decrease clinical contact $(25,29,31,33,40,46,61)$. For instance, Lee, Liem (29) reported that recovery services have improved most outcome parameters such as bed days, re-admission episodes and days of missing psychiatric appointments. Conversely, Malinovsky, Lehrer (39) suggested that the number of days spent in the hospital decreased by about $40 \%$ after initiation of recovery transformation. Furthermore, some studies suggested that the effects of IPS intervention on the time patients spend in competitive employment have had a significant effect on the clinical status, particularly a reduction of the need for psychiatric inpatient care $(31,61)$. For instance, Kilian, Lauber $(61)$ indicated that consumers who received an IPS intervention spent fewer days in the hospital.

\section{Improve Functionality}

Fourteen of the included papers concluded that recovery services have improved the functioning of adults living with severe mental illness (Table 3 ). The recovery services improved the social and psychological functioning as well as motor and process ability of adults living with severe mental illness. More specifically, Asher, Hanlon (46) reported in a study that CBR intervention improved the functioning in adults with schizophrenia (baseline median WHODAS = 57.5, IQR interquartile range 36.7, 65.1 to end line median WHODAS $=18.4$, IQR 2.4,46.2). Similarly, Zhou, Zhou (43) showed in a study that the intervention (eg. rehabilitation training programme such as day treatment, medication monitoring, biweekly rehabilitation training) group had a significant improvement in social and psychological functioning.

\section{Reduce Symptoms}

Most of the included papers suggested that recovery services have had a significant improvement in anxiety, psychosocial and mental symptoms of an adults with severe mental illness $(25,27-30,33,34,40,43,45,61)$. For instance, Chang, Chen (27) reported that the anxiety symptoms in an experimental group (music-creation programme) improved 6.22 points more than the control group $(P<0.001)$. Similarly, the mean symptoms (Positive and Negative Syndrome Scale PANSS) in a clubhouse group (eg. Open occupation or employment) decreased from 64.5 to 42.7 compared with the control groups which increased from 51.7 to $57.6(p=0.01$ ) (30). More importantly, Lopez-Navarro, Del Canto (45) concluded that incorporating mindfulness intervention into integrated rehabilitation has the potential to reduce negative symptoms.

\section{Improvement in Physical health \& Social Behaviour}

Six of the included papers reported that recovery services have improved the physical health and social behaviour of an adults with severe mental illness (26, $33,34,39,45,46)$. In particular, the recovery services have had improvements in physical health, wellbeing, adaptation, appearance, (33, 34, 39, 46), quality of life (psychological health) (45) and reductions in risk-taking behaviour (26). For instance, Lopez-Navarro, Del Canto (45) recommended that incorporating mindfulness-based intervention into recovery services have the ability to improve the psychological health-related quality of life. More specifically, the study indicated that the mindfulness intervention accounts for $38 \%$ of the variance in health-related psychological quality of life (45).

\section{Self-efficacy}

Twenty-one of the included papers described the impact of recovery services on the self-efficacy or self-reliance of adults with severe mental illness (see Table $3)$. The papers highlighted that recovery services have improved self-esteem or self-confidence (eg. fostered feeling of self-worth) (26, 30, $31,38,42,52,57-$ $59)$, hope $(25,27,46,56)$, improvements in thoughts, emotions and better understanding of mental illness $(25,26,31)$. More so, the recovery services have improved self-care or practical skills of daily life (eg. bathing, washing clothes, chores related to food preparation and household cleaning) (26, 56) as well as personal empowerment $(52,56,58,59)$.

Three papers recommended that recovery narrative photovoice and art-making services have positively impacted on adults with severe mental illness. This recovery services have improved the lives of consumers in areas such as senses of identity, independence, tolerating uncertainty, feeling ownership of choices, learning from the past, and maintaining vigilance for relapse (57-59). In particular, art-making interventions provide opportunities for self-exploration and the development of new skills (57). More importantly, the art-making and exhibition help people living with severe mental illness to clearly express their feelings and communicate emotions and thoughts which could be difficult to express using words. In addition, recovery narrative photovoice intervention can also help to achieve recovery outcomes such as autonomy, readiness for change, inspiration, idealism, the transformation of self, acceptance of support, awareness of progress, hope, determination, passion, perseverance, introspection, strength, and sense of connectedness $(58,59)$.

Moreover, four papers recommended that vocational rehabilitation interventions have impacted positively on the personalities (eg. sense of competence through participation in work), reduce boredom/loneliness, feeling anchored in reality and create strong internal motivation for change $(49,52,55,62)$. The feelings and expressions of clients help them to develop a sense of self-determination and personal recovery $(54,62)$.

\section{Economic empowerment}

Nineteen of the included papers reported that the recovery services, for instance, vocational participation have helped to improve the economic empowerment of adult with severe mental illness (see Table 3). More importantly, the vocational rehabilitation programmes provide livelihood and income-generating activities $(26,46,49,52,53)$.

Most papers reported that adult living with severe mental illness who participate in vocational interventions gained competitive employment (28, 33, 37, 50, $53)$, returned to open employment (35) and receive vocational benefit $(37,49,50,63)$. In particular, adult living with severe mental illness participating in 
vocational interventions are more likely to receive job-seeking assistance (eg. searching for jobs, filling application forms and practise for interviews) (38, 49 , 50).

Most of the studies suggested that vocational intervention such as IPS is more effective than the conventional vocational services, particularly in every vocational outcome.The IPS clients are more effective to work competitively, returning to open employment (eg. working for at least one day), and longer duration of employment (eg. working for many hours and longer job tenure) and wages earned $(28,35,48,49,51,61)$. Catty, Lissouba (51) reported that IPS clients were two times (214 days) more likely to work for a longer duration than vocational service clients (108 days). Conversely, $57 \%$ of IPS clients (a sample of 58 consumers with schizophrenia) worked competitively, compared with $29 \%$ of conventional vocational clients. Similarly, $70 \%$ of IPS participants obtained any paid work, compared with $36 \%$ of conventional vocational clients (48). More importantly, the vocational rehabilitation interventions have helped consumers to gain financial literacy skills (eg. managing finances) $(25,26,31,46,52)$, become financial independence and financially stable (49) and improved recovery $(28,46)$.

\section{Social inclusion (Community integration)}

Twenty-seven of the included papers reported that recovery services have increased social inclusion and community acceptance or integration of adults with severe mental illness (see Table 3). Specifically, the recovery services have achieved increasing social or community participation (eg. participating in social activities such as churches, coffee ceremonies weddings and funerals), reduce discrimination $(37,46)$, reduce social isolation, create supportive social environment (25), increase social contacts or social interactions $(26,29,34,37,49,55,60,64)$ and socialization (eg. being around and having breaks and playing games) $(37,60,64)$.

Some studies reported that recovery services such as IPS recovery narrative photovoice and art-making help an adult with severe mental illness to achieve or re-establish valued roles in the community $(31,57,58)$. Whitley, Harris $(42)$ recommended that consumers with severe mental illness can use the community as a place of safety, surrogate family, and as socialization and individual growth. In addition, vocational participation rehabilitation services increase the social contacts of adult with SMI with their supervisors and customers or clients, which subsequently break the feeling of social isolation $(49,52,54,55,64)$.

Furthermore, some studies suggested that the social environment recovery services, (eg. clubhouse used as a community) have the ability to create an atmosphere of acceptance and inclusion and subsequently support each member's personal recovery journey $(54,62)$. For instance, an adult with severe mental illness in a residential programme (clubhouse) had greater participation in recreational events, informal socialization with peers (44), social relationship, quality of life $(30,43)$ and feeling valued, inclusion and belonging to a group (63). Further, De Heer-Wunderink, Visser (32) reported that supported independent living programs seemed to positively influence the level of social inclusion for consumers living with severe mental illness, in terms of their being active and receiving and making visits with others.

\section{Household integration}

Three of the included papers reported that recovery services have achieved integration of adult with severe mental illness into their families $(25,33,46)$. Such recovery services have increased greater participation in the household task and family stability and care $(25,46)$. For instance, Asher, Hanlon $(46)$ reported that a recovery-oriented rehabilitation service has helped an adult with severe mental illness return home to address the basic needs of shelter and food. The services have also equipped family caregivers to develop some resilience to accommodate their relatives, including telling them of plans in advance, communicating calmly and avoiding stressors. Consequently, the service has helped to reduce caregiver burden as well as treating an adult with severe mental illness with dignity and effective caregiving (eg providing food, shelter and shelter) (46).

\section{Social support}

Seven of the included papers highlighted several support services used to implement recovery services for an adult with SMI (28, 31-33, 55, 59). Some papers highlighted that the support services originate from sources such as relatives, friends or peers $(32,55)$ supervisor support and community peers $(31,55)$. In particular, De Heer-Wunderink, Visser (32) suggested that more than $85 \%$ of clients in recovery-oriented rehabilitation service reported having received support from a partner, their family, or friends. Conversely, Harpaz-Rotem, Rosenheck (33) reported that clients receiving a residential treatment had a significantly higher social support on average $(p<0.001)$ after baseline. Supervisors (staff) from recovery services provide practical and emotional support to adults with severe mental illness $(42,55)$. Whitley, Harris $(42)$ reported that most adults with severe mental illness considered supervisors or staff to be equally important members of the surrogate family.

\section{Discussion}

This review was conducted to synthesize evidence into the recovery services used to promote recovery among adults living with severe mental illness. The study specifically aims 1 ) to identify existing recovery services; 2 ) to synthesise evidence on the usefulness of recovery services on the recovery process of consumers. The review findings are discussed according to two emerging themes: (i) philosophy of Recovery services, (ii) usefulness of recovery services on the lives of adults living with a severe mental illness.

\section{Philosophy of Recovery services}

Recovery services are interventions that aim to provide person-centred mental health services. This approach of mental health services employs a whole system or holistic approach towards the recovery journey of consumers (8). The review findings identified several recovery services which include integrated recovery service model, vocational rehabilitation as well as recovery narrative photovoice and art-making exhibition. The integrated recovery service model, for instance, is offered through illness management, mindfulness interventions, task-shifting approach (eg. participatory based training), home visiting, active 
leisure and music therapy services. The Integrated recovery service model are mostly incorporated into conventional services and aim to achieve a holistic mental health services. The review findings encourage service providers to integrate mindfulness practices, active leisure, music therapy and spiritual healing practices as part of the integrated recovery service model $(27,37,45)$. The inclusion of such components not only ameliorate the symptoms but are also useful in achieving a sense of agency and autonomy, taking personal responsibility and getting on with life (10). Conversely, the integrated recovery service model encourages the use of task-shifting and home visiting to promote the recovery journey $(25,26,29,46,47)$. The home visiting and task-shifting can help consumers to take up a central role in managing illness (eg. regaining control), personal growth as well as establishing a fulfilling and meaningful life (3). The task-shifting and home visiting can help consumers and their families to set their personal goals towards the recovery journey. Our review findings encourage service providers to implement the integrated recovery service models to promote the personal recovery process of consumers.

The evidence suggests that vocational rehabilitation services are also increasingly employed to promote the recovery process of consumers This service is mostly offered through IPS, supported employment enterprises and social firms compared with conventional vocational services (eg. sheltered employment) $(35,51)$. The vocational rehabilitation services that are used to support the recovery process of consumers are consistent with previous literature (12-14). In particular, the IPS interventions have proven to have the ability to improve the recovery journey. The IPS interventions identified in the current findings are mostly associated with agricultural production, creative projects, services and clients joining the labour market $(30,35,49,51)$. The interventions are usually implemented through initial vocational assessment, job searching, individual job development, monitoring work performance, support for employers and continuing post-employment support for clients. The review findings encourage service providers to implement vocational rehabilitation services (eg IPS interventions) that are contextually respected by local service providers and communities. More specifically, researchers are encouraged to use interventional studies to measure the effectiveness of the different vocational rehabilitation services that aim to promote the recovery process of consumers.

Recovery narrative photovoice, art-making and exhibition interventions have recently been employed as recovery services to support the recovery process (5660 ). The photovoice, art-making and exhibition services are implemented through text construction and photographs. This service is also presented through an exhibition and large group discussion. The service is particularly employed to achieve recovery, empowerment, community integration, expressing difficult experiences in non-verbal forms as well as avoiding the stigma associated with conventional mental health services. The review findings encourage researchers to use interventional studies to explore the effectiveness of recovery narrative photovoice and art-making exhibition services towards the recovery journey, particularly in multi-cultural settings, where there is increase stigmatization of mental illness. Such interventional studies could help consumers to develop culturally sensitive recovery goals.

\section{Usefulness of recovery services}

Recovery services necessarily focus on enhancing consumer's capacities for living with, managing, and pursuing a life in the presence of disability, as well as removing barriers around their environment (4). Consistent with earlier studies, the current recovery services are useful in enhancing psychiatric medication and treatment (clinical outcomes) of consumers $(4,6)$. More specifically, such services increase access to psychiatric medication, antipsychotic medication adherence, decrease relapse, improve knowledge and decrease clinical contact $(25,29,31,33,40,46,61)$. These services are not only about helping consumers to learn how to live a fuller and more satisfying life but also contributes to the reduction of the symptom itself. The review findings encourage service providers to promote holistic care that considers the individual's subjective appraisal of his or her functioning and satisfaction with life (9).

In addition, psychiatric rehabilitation has recently moved beyond the mere control of symptoms and prevention of relapse to incorporate a functional recovery and enhancement of the quality of life of the consumer (3). The ability of service providers to improve the quality of life of consumers could help to achieve a personal recovery process that is consumer-centred. The current review findings demonstrated that recovery services have supported the physical health and social behaviour of consumers. More specifically, such recovery services improves the physical health, well-being, adaptation, appearance, quality of life and reduction in risk-taking behaviour $(26,33,34,39,45,46)$. The improvement in the physical and social behaviour of the consumers could also help them to progress in developing self-efficacy, self-confidence, and gaining hope, improvements in self-care or practical skills. The interventions have specifically enhanced the recovery process through readiness for change, autonomy, inspiration, idealism, sense of connectedness and transformation of self.

Recovery services have also improved the economic empowerment of adult living with severe mental illness. The services provide livelihood and incomegenerating avenues that can facilitate access to competitive employment, returning to open employment and vocational benefits. It is apparent that the participation in income generation activities improves the financial literacy skills, financial independence and financial stability of adult living with severe mental illness. The improvement in finances through income-generating activities forms a major component of the recovery journey or process. The findings confirm previous literature which suggests that recovery services could empower adults with severe mental illness, through normative life participation such as education, social and political activities $(3,5)$. The major strength of recovery services mostly relies on its ability to safeguard empowerment in the consumers through everyday living skills, accommodation, social networks, employment and education endeavours (5). Again, the review findings demonstrated that recovery services have the ability to aid the social inclusion and community acceptance of adult living with severe mental illness. Consistent with previous literature, recovery-oriented rehabilitation promotes the inclusion of adults with severe mental illness through increasing community participation, social contacts or social interactions, socialization, and supportive social environment (6). Such services support consumers to reconnect and reestablish a place in the community, and to explore opportunities that could help them live an independent life. Consequently, the services reduce social isolation, discrimination and stigmatization among consumers (5). In addition, the consumers are also integrated greatly into their individual families and so take an active role in family activities and also maintain family stability.

\section{Limitations}


The review has several limitations that need consideration. The limitations of the integrative review are largely pertinent to the search words, language limitations, and period of included papers. The review was only limited to papers published in English Language and those published from January 2008 to January 2020. In particular, limiting studies to only English Language articles published between January 2008 to January 2020 could miss relevant nonEnglish Language articles as well as those published before 2008. The variation in search terms and keywords regarding recovery-oriented rehabilitation and recovery based interventions may miss some relevant articles. However, the combination of clearly articulated search methods, consultation with research Librarian, and reviewing articles with multiple experts as well as the quality assessment tool used to measure the methodological quality helped to address the various limitations.

\section{Conclusion}

The review findings showed that several studies have been undertaken regarding recovery services that can facilitate the recovery journey for adults living with severe mental illness. Most of the recovery services are implemented in developed western countries, particularly in the USA and Europe, with relatively few studies piloted in developing countries (for example Africa). The review findings demonstrated that most of the papers used quantitative data, with few studies employing both qualitative and quantitative data to achieve complementarity or convergence. The evidence showed that most recovery services are delivered through community-based settings. Also, studies on recovery services largely address issues on integrated recovery service model and vocational rehabilitation, with few studies addressing recovery narrative photovoice and art-making exhibition services. Furthermore, the recovery services are reported to be useful in areas such medication and treatment adherence, functioning, symptoms, physical health \& social behaviour, self-efficacy, economic empowerment, social inclusion or community integration, household integration and increase access to social support services.

\section{Relevance for Clinical Practice, Policy and Future Research}

- The recovery services identified are valuable for Mental Health Professionals. These findings may lead to an adaptation of rehabilitation interventions that would be helpful for the personal recovery goals for consumers.

- Awareness and advocacy for recovery recovery services should be prioritised in national and international policy initiatives. Consumer associations, selfhelp groups and family caregivers could be empowered to take the leading advocacy role in the recovery services.

- Research on recovery services should be prioritized in clinical practice and further directed towards interventional studies, which can provide sustainable and workable solutions in the recovery journey and outcome.

- Recovery research mostly employs quantitative and qualitative methods, with relatively few studies using mixed methods. We recommend that future recovery research should attempt to use mixed methods to achieve complementarity and congruence in both methods.

\section{List Of Abbreviations}

Individual Placement Services (IPS); Joanna Briggs Institute (JBI); Mixed Methods Appraisal Tool (MMAT); Preferred Reporting Items for Systematic Reviews and Meta-Analyses (PRISMA); Severe Mental illness (SMI); World Health Organization Disability Assessment Scale (WHODAS)

\section{Declarations}

\section{Ethics approval and consent to participate}

Not applicable

\section{Consent for publication}

Not applicable

\section{Availability of data and materials}

All data generated or analysed during this study are included in this article and its supplementary information files

\section{Competing interests}

The authors declare that they have no competing interests.

\section{Funding}

This research did not receive any specific grant from funding agencies in the public, commercial, or not-for-profit sectors.

\section{Authors' contributions}

E.B, A.P.O, and R.M conceptualized the study. E.B conducted the data extraction, A.P.O, and R.M, conducted the second review of the extracted data. E.B, working closely with A.P.O and R.M performed the thematic analysis and drafted the manuscript. All authors read and approved the manuscript and made inputs into the intellectual content and agreed on its submission for publication.

\section{Acknowledgements}


The authors wish to thank the University of Newcastle Graduate Research (Australia), for the Doctoral Scholarship offered to the lead author.

\section{References}

1. Roessler W. Psychiatric rehabilitation today: an overview. World Psychiatry. 2006;5(3):151.

2. Killaspy H, Harden C, Holloway F, King M. What do mental health rehabilitation services do and what are they for? A national survey in England. J Ment Health. 2005;14(2):157-65.

3. Yildiz M. Recovery as a process in severe mental illnesses. Nöro Psikiyatri Arşivi. 2015;52(1):1.

4. Davidson L, Roe D. Recovery from versus recovery in serious mental illness: One strategy for lessening confusion plaguing recovery. J Ment Health. 2007;16(4):459-70.

5. Frost BG, Tirupati S, Johnston S, Turrell M, Lewin TJ, Sly KA, et al. An Integrated Recovery-oriented Model (IRM) for mental health services: evolution and challenges. BMC Psychiatry. 2017;17.

6. Gopal S, Henderson AR. Trans-cultural study of recovery from severe enduring mental illness in Chennai, India and Perth, Western Australia. Journal of Psychosocial Rehabilitation and Mental Health. 2015;2(1):51-7.

7. Slade M, Longden E. Empirical evidence about recovery and mental health. BMC Psychiatry. 2015;15(1):285.

8. Drake RE, Whitley R. Recovery and Severe Mental IIIness: Description and Analysis. Canadian Journal of Psychiatry-Revue Canadienne De Psychiatrie. 2014;59(5):236-42.

9. Psych B, Remington G, Lee J. Personal recovery in serious mental illness: Making sense of the concept. Ann Acad Med Singapore. 2017;46:29-31.

10. Noordsy D, Torrey W, Mueser K, Mead S, O'Keefe C, Fox L. Recovery from severe mental illness: an intrapersonal and functional outcome definition. International Review of Psychiatry. 2002;14(4):318-26.

11. Badu E, O'Brien AP, Mitchell R. The Conceptualization of Mental Health Service Quality Assessment: Consumer Perspective. Administration and Policy in Mental Health and Mental Health Services Research. 2019;26(6):790-806.

12. Gibson RW, D'Amico M, Jaffe L, Arbesman M. Occupational Therapy Interventions for Recovery in the Areas of Community Integration and Normative Life Roles for Adults With Serious Mental Illness: A Systematic Review. American Journal of Occupational Therapy. 2011;65(3):247-56.

13. Metcalfe JD, Drake RE, Bond GR. Economic, Labor, and Regulatory Moderators of the Effect of Individual Placement and Support Among People With Severe Mental Illness: A Systematic Review and Meta-analysis. Schizophrenia Bulletin. 2018;44(1):22-31.

14. Gmitroski T, Bradley C, Heinemann L, Liu G, Blanchard P, Beck C, et al. Barriers and facilitators to employment for young adults with mental illness: a scoping review. Bmj Open. 2018;8(12).

15. Whittemore R, Knafl K. The integrative review: updated methodology. Journal of advanced nursing. 2005;52(5):546-53.

16. Hopia H, Latvala E, Liimatainen L. Reviewing the methodology of an integrative review. Scandinavian Journal of Caring Sciences. 2016;30(4):662-9.

17. National Institute of Mental Health. Mental Illness USA: U.S. Department of Health and Human Services; 2018 [cited 2019 September 2, ]. Available from: https://www.nimh.nih.gov/health/statistics/mental-illness.shtml.

18. Pratt LA. Characteristics of adults with serious mental illness in the United States household population in 2007. Psychiatr Serv. 2012;63(10):1042-6.

19. Pearson A, White H, Bath-Hextall F, Apostolo J, Salmond S, Kirkpatrick P. Methodology for JBI mixed methods systematic reviews. The Joanna Briggs Institute Reviewers Manual. 2014;1:5-34.

20. Moher D, Liberati A, Tetzlaff J, Altman DG, Group P. Preferred reporting items for systematic reviews and meta-analyses: the PRISMA statement. PLoS medicine. 2009;6(7):e1000097.

21. Hong N. Quan, Pluyr P, Fabregues S, Bartlett G, Boardman F, Cargo M, et al. Mixed Methods Appraisal Tool (MMAT). Canada.: Intellectual Property Office, Canada; 2018.

22. The Joanna Briggs Institute (JBI). Critical Appraisal Tools Australia: The University of Adelaide; 2017 [Available from: http://joannabriggs.org/research/critical-appraisal-tools.html.

23. Braun V, Clarke V. Using thematic analysis in psychology. Qualitative research in psychology. 2006;3(2):77-101.

24. Fossey E, Harvey C, McDermott F, Davidson L. Understanding and evaluating qualitative research. Australian and New Zealand Journal of Psychiatry. 2002;36(6):717-32.

25. Brooke-Sumner C, Lund C, Selohilwe O, Petersen I. Community-based psychosocial rehabilitation for schizophrenia service users in the north west province of South Africa: A formative study. Social Work in Mental Health. 2017;15(3):249-83.

26. Brooke-Sumner C, Selohilwe O, Mazibuko MS, Petersen I. Process Evaluation of a Pilot Intervention for Psychosocial Rehabilitation for Service Users with Schizophrenia in North West Province, South Africa. Community Ment Health J. 2018;54(7):1089-96.

27. Chang BH, Chen BW, Beckstead JW, Yang CY. Effects of a music-creation programme on the anxiety, self-esteem, and quality of life of people with severe mental illness: A quasi-experimental design. International Journal of Mental Health Nursing. 2018;27(3):1066-76.

28. Chiu MYL, Ho WWN, Lo WTL, Yiu MGC. Operationalization of the SAMHSA model of recovery: a quality of life perspective. Qual Life Res. 2010;19(1):1-13.

29. Lee CC, Liem SK, Leung J, Young V, Wu K, Kenny KKW, et al. From deinstitutionalization to recovery-oriented assertive community treatment in Hong Kong: What we have achieved. Psychiatry Res. 2015;228(3):243-50.

30. Tsang AW, Ng RM, Yip K. A six-month prospective case-controlled study of the effects of the clubhouse rehabilitation model on Chinese patients with chronic schizophrenia. East Asian Archives of Psychiatry. 2010;20(1):23.

Page 20/22 
31. Crain M, Penhale C, Newstead C, Thomson L, Heah T, Barclay K. The contribution of IPS to recovery from serious mental illness: A case study. Work-a Journal of Prevention Assessment \& Rehabilitation. 2009;33(4):459-64.

32. De Heer-Wunderink C, Visser E, Sytema S, Wiersma D. Social inclusion of people with severe mental illness living in community housing programs. Psychiatr Serv. 2012;63(11):1102-7.

33. Harpaz-Rotem I, Rosenheck RA, Desai R. Residential treatment for homeless female veterans with psychiatric and substance use disorders: Effect on 1year clinical outcomes. Journal of Rehabilitation Research and Development. 2011;48(8):891-900.

34. Lindstrom M, Hariz GM, Bernspang B. Dealing With Real-Life Challenges: Outcome of a Home-Based Occupational Therapy Intervention for People With Severe Psychiatric Disability. Otjr-Occupation Participation and Health. 2012;32(2):5-13.

35. Burns T, White SJ, Catty J, Grp E. Individual Placement and Support in Europe: The EQOLISE trial. International Review of Psychiatry. 2008;20(6):498-502.

36. Hultqvist J, Markstrom U, Tjornstrand C, Eklund M. Quality of life among people with psychiatric disabilities attending community-based day centres or Clubhouses. Scandinavian Journal of Caring Sciences. 2018;32(4):1418-27.

37. Tjornstrand C, Bejerholm U, Eklund M. Participation in day centres for people with psychiatric disabilities: Characteristics of occupations. Scandinavian Journal of Occupational Therapy. 2011;18(4):243-53.

38. Luk AL. Investigating the long-term effects of a psychiatric rehabilitation programme for persons with serious mental illness in the community: A followup study. J Clin Nurs. 2011;20(19-20):2712-20.

39. Malinovsky I, Lehrer P, Silverstein SM, Shankman SA, O'Brien W, Samuelson T, et al. An empirical evaluation of recovery transformation at a large community psychiatric rehabilitation organization. Psychological Services. 2013;10(4):428-41.

40. Salyers MP, McGuire AB, Kukla M, Fukui S, Lysaker PH, Mueser KT. A Randomized Controlled Trial of Illness Management and Recovery With an Active Control Group. Psychiatr Serv. 2014;65(8):1005-11.

41. Tondora J, O'Connell M, Miller R, Dinzeo T, Bellamy C, Andres-Hyman R, et al. A clinical trial of peer-based culturally responsive person-centered care for psychosis for African Americans and Latinos. Clinical Trials. 2010;7(4):368-79.

42. Whitley R, Harris M, Fallot RD, Berley RW. The active ingredients of intentional recovery communities: Focus group evaluation. J Ment Health. 2008;17(2):173-82.

43. Zhou Y, Zhou R, Li W, Lin Y, Yao J, Chen J, et al. Controlled trial of the effectiveness of community rehabilitation for patients with schizophrenia in Shanghai, China. Shanghai Archives of Psychiatry. 2015;27(3):167-74.

44. Zemore SE, Kaskutas LA. Services received and treatment outcomes in day-hospital and residential programs. J Subst Abuse Treat. 2008;35(3):232-44.

45. Lopez-Navarro E, Del Canto C, Belber M, Mayol A, Fernandez-Alonso O, Lluis J, et al. Mindfulness improves psychological quality of life in communitybased patients with severe mental health problems: A pilot randomized clinical trial. Schizophrenia Research. 2015;168(1-2):530-6.

46. Asher L, Hanlon C, Birhane R, Habtamu A, Eaton J, Weiss HA, et al. Community-based rehabilitation intervention for people with schizophrenia in Ethiopia (RISE): a 12 month mixed methods pilot study. BMC Psychiatry. 2018;18.

47. Iwasaki Y, Coyle C, Shank J, Messina E, Porter H. Leisure-Generated Meanings and Active Living for Persons With Mental Illness. Rehabilitation Counseling Bulletin. 2013;57(1):46-56.

48. Twamley EW, Vella L, Burton CZ, Becker DR, Bell MD, Jeste DV. The efficacy of supported employment for middle-aged and older people with schizophrenia. Schizophrenia Research. 2012;135(1-3):100-4.

49. Koletsi M, Niersman A, van Busschbach JT, Catty J, Becker T, Burns T, et al. Working with mental health problems: clients' experiences of IPS, vocational rehabilitation and employment. Social Psychiatry and Psychiatric Epidemiology. 2009;44(11):961-70.

50. Waghorn G, Dias S, Gladman B, Harris M. Measuring what matters: Effectiveness of implementing evidence-based supported employment for adults with severe mental illness. International Journal of Therapy and Rehabilitation. 2015;22(9):411-20.

51. Catty J, Lissouba P, White S, Becker T, Drake RE, Fioritti A, et al. Predictors of employment for people with severe mental illness: results of an international six-centre randomised controlled trial. Br J Psychiatry. 2008;192(3):224-31.

52. Panczak A, Pietkiewicz I. Work activity in the process of recovery- an interpretive phenomenological analysis of the experiences of people with a schizophrenia spectrum diagnosis. Psychiatr Pol. 2016;50(4):805-26.

53. Browne DJ, Waghorn G. Employment services as an early intervention for young people with mental illness. Early Intervention in Psychiatry. 2010;4(4):327-35.

54. Svanberg J, Gumley A, Wilson A. How do Social Firms Contribute to Recovery from Mental Illness? A Qualitative Study. Clinical Psychology \& Psychotherapy. 2010;17(6):482-96.

55. lancu SC, Zweekhorst MBM, Veltman DJ, van Balkom A, Bunders JFG. Mental health recovery on care farms and day centres: a qualitative comparative study of users' perspectives. Disability and Rehabilitation. 2014;36(7):573-83.

56. Clements K. Participatory action research and photovoice in a psychiatric nursing/clubhouse collaboration exploring recovery narrative. J Psychiatr Ment Health Nurs. 2012;19(9):785-91.

57. Fenner P, Ryan B, Latai L, Percival S. Art making and the promotion of wellbeing in Samoa - participants' lived experience of a recovery oriented intervention. Arts \& Health. 2018;10(2):124-37.

58. Mizock L, Russinova Z, DeCastro S. Recovery Narrative Photovoice: Feasibility of a Writing and Photography Intervention for Serious Mental Illnesses. Psychiatr Rehabil J. 2015;38(3):279-82. 
59. Mizock L, Russinova Z, Shani R. New roads paved on losses: Photovoice perspectives about recovery from mental illness. Qualitative Health Research. 2014;24(11):1481-91.

60. Ketch RA, Rubin RT, Baker MR, Sones AC, Ames D. Art appreciation for veterans with severe mental illness in a VA Psychosocial Rehabilitation and Recovery Center. Arts \& Health. 2015;7(2):172-81.

61. Kilian R, Lauber C, Kalkan R, Dorn W, Rossler W, Wiersma D, et al. The relationships between employment, clinical status, and psychiatric hospitalisation in patients with schizophrenia receiving either IPS or a conventional vocational rehabilitation programme. Social Psychiatry and Psychiatric Epidemiology. 2012;47(9):1381-9.

62. Raeburn T, Schmied V, Hungerford C, Cleary M. The use of social environment in a psychosocial clubhouse to facilitate recovery-oriented practice. Bjpsych Open. 2016;2(2):173-8.

63. Hultqvist J, Markström U, Tjörnstrand C, Eklund M. Programme characteristics and everyday occupations in day centres and clubhouses in Sweden. Scandinavian journal of occupational therapy. 2017;24(3):197-207.

64. Swildens W, van Busschbach JT, Michon H, Kroon H, Koeter MWJ, Wiersma D, et al. Effectively Working on Rehabilitation Goals: 24-Month Outcome of a Randomized Controlled Trial of the Boston Psychiatric Rehabilitation Approach. Canadian Journal of Psychiatry-Revue Canadienne De Psychiatrie. 2011;56(12):751-60.

\section{Figures}

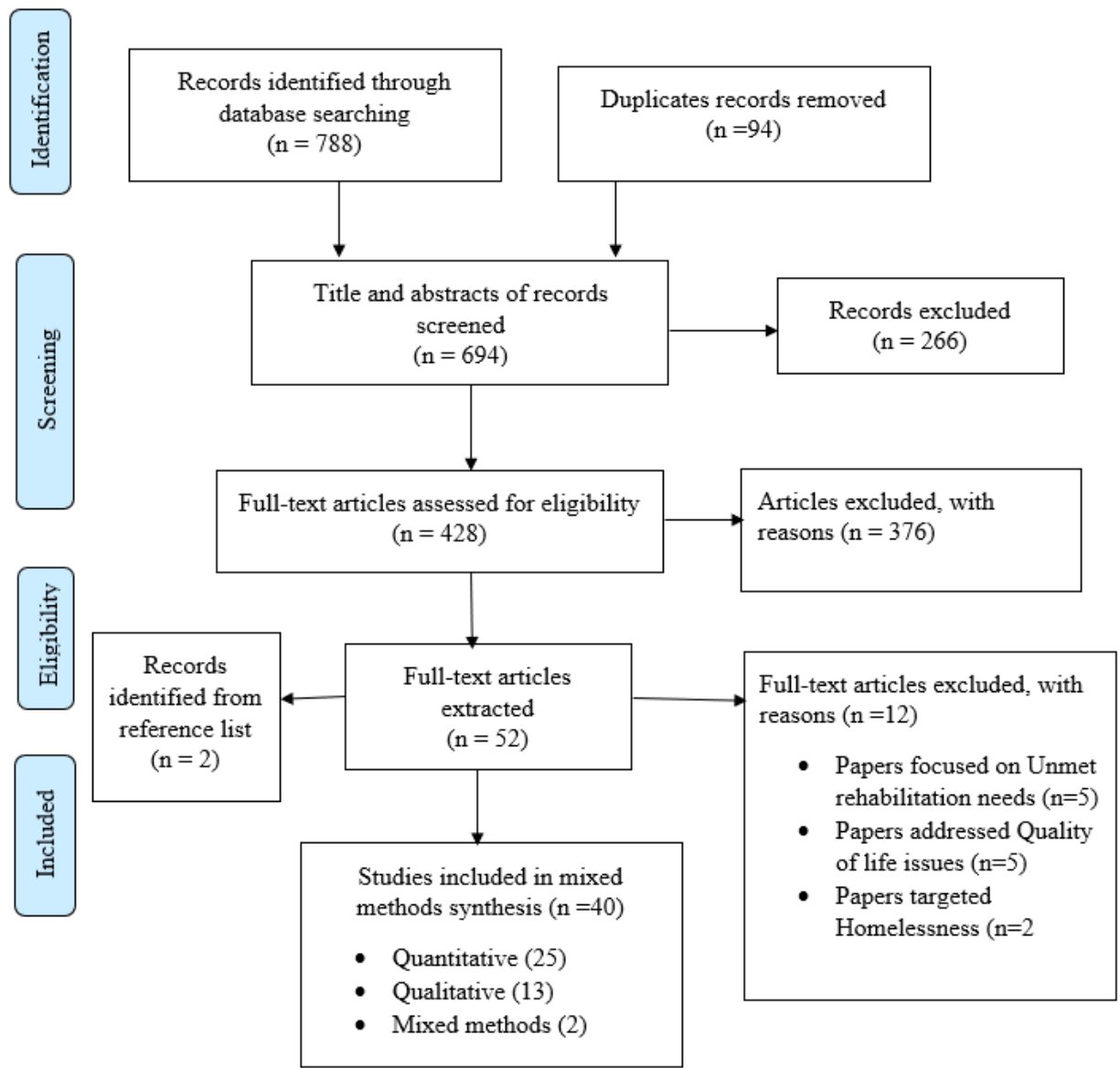

\section{Figure 1}

Flow chart of included papers

\section{Supplementary Files}

This is a list of supplementary files associated with this preprint. Click to download.

- AdditonalFile3PRISMA2009checklist.pdf

- AdditionalFile2MethodologicalQualityAssessment.pdf

- AdditionalFile1Dataextraction.pdf 